\title{
IMPLEMENTATION OF INJURY AND ILLNESS SURVEILLANCE PROTOCOLS IN VARSITY ATHLETES
}

By

ALEXANDER S. D. GAMBLE

\author{
A Thesis \\ Presented to \\ The University of Guelph
}

In partial fulfillment of requirements

for the degree of:

Masters of Science (MSc)

in

Human Health and Nutritional Sciences

Guelph, Ontario, Canada

(C) Alexander S. D. Gamble, April 2017 


\begin{abstract}
IMPLEMENTATION OF INJURY AND ILLNESS SURVEILLANCE PROTOCOLS IN VARSITY ATHLETES.
\end{abstract}

Alexander Shand Davis Gamble

University of Guelph, 2017
Advisor:

Professor Lawrence L. Spriet

This thesis is involves the assessment of a varsity athlete injury and illness surveillance system that will strengthen and standardize the quality of the data collected, and support the development of sport-specific preventative measures for future athletes. Injury and illness information was collected from 14 varsity teams (338 varsity athletes) from the University of Guelph during the Fall 2016 semester season. Descriptive data collected involved weekly summaries of injuries and illnesses and individual reporting on each injury and illness completed by athletes and team trainers. The average compliance from varsity teams was $86 \%$ (men: $\sim 96 \%$, women: $\sim 72 \%$ ). Injury prevalence was 9.0 (95\% CI 7.3 to 10.6) injuries per 1000 athlete exposures and illness prevalence was 9.2 illnesses (95\% CI 6.1 to 12.3 ) per 100 participating athletes. Athlete injury and illness surveillance was feasible in Canadian university sports and will help develop prevention strategies in future competitive seasons. 


\section{ACKNOWLEDGEMENTS}

To be honest, it is hard to know where to start. I have been so lucky to have a great support system throughout my entire education, and my life for that matter. First off, I have to thank all of the athletes, team trainers, medical staff, and others that were involved in my Masters project. There are far too many names to list but this research wouldn't have been possible without them. I appreciate all of them putting their trust in our group of researchers, and hope that we will be able to positively influence university sport and athletes with our findings. This was always our first goal.

I have to thank my family for everything. There were a few times when I went home during undergrad not knowing if I was going to make it, and now I have successfully completed my Masters. There is no way I would have been able to make it this far without all of their support. My parents, Karen and Mark, have been wonderful and were always there for me to vent when I was "a tad" stressed out. They were able to push me so that I worked as hard as I could, but were also able to take away unneeded pressure by making me feel like everything would be fine if something didn't work out. My sister Raven was always there to listen to my crazy stories (not always education related), which also gave me another line to shout down if needed. Not only this, but her constant impressive marks in school always helped push me. My grandparents have always been so excited to hear about what I am doing at school and constantly telling me how proud they are of me. Hearing this means the world to me and always helped motivate me a little bit more.

Most of my friends from my hometown have been finished school for a while now, but always kindly ask how I am making out with everything. They are always there when I go home and are happy to celebrate any occasion to help blow off some steam. Throughout 
undergrad, I made some great friends that were awesome to spend time with and together we all learned how to make it through university on a positive note, with quite a bit of fun in between. The friends I have met during my Masters have been nothing short of amazing. I would have to say that my friends Adam, Jess, and Tyler are now my closest friends. Tyler and I have been through the thick of it together since day one and there is no way I could have made it through this process without him. I met Jess in third year of undergrad and we have been great friends ever since. Also, we have to be two of the biggest Leaf's fans ever! She played a pivotal role in being able to successfully complete my Masters project. I am very lucky that we can work together so well, and her help and support through it all made a huge difference. I am very appreciative of developing these strong friendships. I would also like to thank all of my lab mates (Greg, Stephanie, Devin, Kate, Sebastian, Jamie, Tara, Dan, and Owen) for all of their support and friendship over the last two years.

Another person I have to mention is someone who is very special to me. After meeting Brittany Carter in third year, she was there for me throughout my education. I truly don't think that I would be where I am today without her support. Being able to work through things together while having her cheer me on was a lifesaver, especially during those stressful moments throughout university. Spending time together was always a good break from reality and a huge difference-maker for me. To be honest, I will always look up to her and be thankful for all of our experiences together.

I met Margo in August of 2016 and I am very glad that I had the chance to do so. As soon as I heard about this project and who I would be working with, I jumped at the chance. Margo has to be one of the most motivated people I have ever met and it has rubbed off on me while working alongside her on this project. There were a few "speed 
bumps" along the way that we were able to overcome together and I have to thank her for all of her help this year. I constantly look up to her, hoping to become half the professional she is one day. I am looking forward to trying to publish this research with her and hopefully there will be many more projects we can work on together moving forward.

Now back to third year of undergrad when I saw Dr. Lawrence Spriet walking across campus. At this point I knew who he was but I knew nothing about what an amazing person he was. For some reason, I had the urge to follow him back to his office and introduce myself. Assuming the conversation would last a few minutes, I ended up walking out of his office about an hour later after chatting about hockey and everything else under the sun. For some reason, this crazy man gave me a shot and hired me in his lab for the summer of third year. During the semester I met Lawrence, I honestly still wasn't enjoying university very much and was considering other options. When this opportunity came up, I thought I had nothing to lose and I was definitely right. Since then, I have been lucky enough to work in his lab and he has taught me just about everything I know when it comes to the many areas within exercise science. We have had some great experiences together travelling and working with different hockey teams and I genuinely hope this continues in the years to come. I am very thankful for the chance that Lawrence gave me because it has truly changed my life forever.

I have been more than lucky to have all of these people in my life and I will never forget how much they have helped guide me through this journey. I am eternally grateful! 


\section{TABLE OF CONTENTS}

LIST OF ABBREVIATIONS IX IX

LIST OF FIGURES X X

LIST OF TABLES XI

CHAPTER 1: REVIEW OF THE LITERATURE 1

Importance of athlete injury and illness surveillance in sport $\quad 2$

Injury and illness surveillance protocols in elite sport $\quad \mathbf{4}$

Injury surveillance in high school sports

Injury surveillance at the collegiate and university level

Injury surveillance in professional sport

Injury and illness surveillance during FINA World Championships \& Olympic Games

Epidemiology of injuries and illnesses in sports

11

Men's baseball

Men's and women's cross country

Women's field hockey

Men's football

Men's and women's golf

Men's and women's lacrosse

Men's and women's rowing

Men's rugby

Men's and women's soccer

Feasibility and implementation of injury and illness surveillance protocols $\quad \mathbf{2 0}$

"Injury" and "illness" definitions in FINA and IOC prospective athlete surveillance

Compliance and acceptability of injury and illness surveillance

Methodological considerations for controlled injury and illness surveillance

Conclusion

25

CHAPTER 2: AIMS OF THE THESIS

Problem

Purpose

28

Hypotheses 
Subjects $\quad \mathbf{3 0}$

$\begin{array}{ll}\text { Study design } & \mathbf{3 1}\end{array}$

Defining injury and illness, and team trainers

Team trainers

Implementation

Measurements and experimental protocol

Weekly summary of injuries and illnesses reporting form

Athletic therapist/student trainer injury and illness reporting form

Athlete injury and illness reporting form

Data analysis

CHAPTER 4: RESULTS $\quad 40$

Compliance and rate of response $\quad 40$

Prevalence of injuries $\quad 41$

Location, type, and mechanism of injuries $\quad \mathbf{4 4}$

Severity of injuries $\quad \mathbf{5 0}$

Treatment of injuries

Prevalence of illnesses $\quad \mathbf{5 4}$

Affected body system and diagnosis of illnesses $\quad \mathbf{5 6}$

Severity of illnesses $\quad \mathbf{5 7}$

Treatment of illnesses $\quad \mathbf{5 8}$

CHAPTER 5: DISCUSSION

Overview $\quad \mathbf{5 9}$

$\begin{array}{ll}\text { Injury rates in collegiate and university sport } & \mathbf{6 0}\end{array}$

Location, type, and mechanism of injuries $\quad 64$

Severity of injuries $\quad 66$

$\begin{array}{ll}\text { Illness risk in Canadian university sport } & \mathbf{6 8}\end{array}$

Methodological considerations and practical implications $\quad \mathbf{6 8}$

Limitations $\quad \mathbf{7 0}$

$\begin{array}{ll}\text { Conclusion } & \mathbf{7 2}\end{array}$

REFERENCES 
APPENDIX 1: Athletic Therapists/Student Trainers Weekly Report $\quad \mathbf{8 1}$

APPENDIX 2: Athletic Therapists'/Student Trainers' Individual Injury and $\mathbf{8 3}$ Illness Report

APPENDIX 3: Athletes' Daily Report on Injuries and Illnesses 


\section{LIST OF ABBREVIATIONS}

AE Athlete Exposures

AHMS Athlete Health Management System

ANOVA One-way Analysis of Variance

AT Athletic Therapy

CAT Certified Athletic Therapists/Trainer

CI Confidence Interval

CIS Canadian Interuniversity Sport

CISIR Canadian Intercollegiate Sport Injury Registry

EMR Electronic Medical Record

FIFA Fédération Internationale de Football Association

FINA Fédération Internationale de natation

FIVB Federation International de Volleyball

HITS Health and Injury Tracking System

IAAF International Association of Athletics Federations

IHF International Handball Federation

IOC International Olympic Committee

ISS Injury Surveillance System

MCL Medial Collateral Ligament

MLB Major League Baseball

NATA National Athletic Therapists Association

NATION National Athletic Treatment, Injury and Outcomes Network

NBA National Basketball Association

NCAA National Collegiate Athletic Association

NFL National Football League

NFLISS National Football League Injury Surveillance System

NHL National Hockey League

OUA Ontario University Association

RIO Reporting Information Online

RR Risk Ratio

USA United States of America

WR World Rugby 


\section{LIST OF FIGURES}

Figure 1: van Mechelen's model of injury prevention.

Figure 2: Weekly report that team trainers filled out when an athlete was injured.

Figure 3: Weekly report that team trainers filled out when an athlete was ill.

Figure 4: Exposure in training section of team trainers' report.

Figure 5: Exposure in competition section of team trainers' report.

Figure 6: Section of team trainers' report that is answered to provide information on the team trainer's perceived severity of the injury or illness.

Figure 7: Section of team trainers' report that is completed to provide information on the athlete's complaints and missed training caused by the injury or illness, as well as whether the injury or illness was new, recurrent, or reported in previously.

Figure 8: Section of team trainers' report that is completed to provide information on any treatment the athlete received for the injury or illness.

Figure 9: Section of athletes' report that is answered to provide information on the athlete's perceived severity of the injury or illness.

Figure 10: Section of athletes' report that is completed to provide information on their complaints and missed training caused by the injury or illness, as well as whether the injury or illness was new, recurrent, or reported in previously.

Figure 11: Section of athletes' report that is completed to provide information on the location of the complaints caused by their injury or illness.

Figure 12: Section of athletes' report that is completed to provide information on the cause or type of complaints caused by their injury or illness.

Figure 13: Section of athletes' report that is completed to provide information on any diagnosis of their injury or illness.

Figure 14: Compliance, and rate of response from team trainers and athletes for implemented varsity athlete injury and illness surveillance protocol for men and women.

Figure 15: Injury rates of varsity athletes for all teams, men (M), women (W), and athletes

Figure 16: Average injury severity questions completed by athletes and team trainers.

Figure 17: Illness rates of varsity athletes for all teams, men (M), women (W), and athletes. 


\section{LIST OF TABLES}

Table 1: Number of athletes observed during their competition seasons, and number of injuries, illnesses, competition and training athlete exposures (AE), and lengths of season for each varsity sports team.

Table 2: Injury location and type, and mechanism of injury during men's varsity competition.

Table 3: Injury location and type, and mechanism of injury during men's varsity training.

Table 4: Injury location and type, and mechanism of injury during women's varsity competition.

Table 5: Injury location and type, and mechanism of injury during women's varsity training.

Table 6: Men's and women's injury severity represented by training and competition days missed.

Table 7: Treatment of injuries for men and women varsity athletes reported by team trainers.

Table 8: Men's and women's affected body system and diagnosis of illnesses during their competition season.

Table 9: Treatment of illnesses for men and women varsity athletes reported by team trainers. 


\section{CHAPTER 1: REVIEW OF THE LITERATURE}

Advancing performance in today's elite athletes is a popular topic of research for the athletes themselves, their coaches, and especially the medical staff responsible for their health. Over the past decade, research regarding an athlete's optimal diet, their sportspecific training, and the use of ergogenic aids have been a few of the paths taken by sport science researchers. Another trending area of research revolves around the steps that medical personnel take to promote athlete health so they can compete at their best level. According to Finch and colleagues (1999), athlete injury surveillance during sports should be part of the "duty of care" so that sports-specific precautions are taken to avoid causes of negative health for athletes both during their sporting career and in the future following retirement from sport. In addition, without medical professionals expending efforts to ensure the health of their athletes, sport performances will decrease.

The objective of this literature review is to explain the implementation and application of athlete injury and illness surveillance protocols in sport across various levels of experience and competition. It aims to identify previous and current implementations of athlete injury and illness surveillance, and their compliance and acceptability in sport. By discussing the accepted definitions of "injury" and "illness", it should also clarify the importance of controlled definitions when collecting this data (Junge et al. 2008b, Ljungqvist et al. 2009, Mountjoy et al. 2015, Prien et al. 2016). Injury epidemiology will also be identified for men's baseball, men's and women's cross country, women's field hockey, men's football, men's and women's golf, men's and women's lacrosse, men's and women's rowing, men's rugby, and men's and women's soccer to pave the way for thoughts on mechanisms of injury in these sports. Following van Mechelen's model of injury prevention, 
these are the first steps toward assessing updated sport-specific prevention measures for athletes in the future (van Mechelen et al. 1992).

\section{Importance of athlete injury and illness surveillance in sport}

Many of the reasons for executing this research revolve around the Olympic Charter's Fundamental Principles and Regulations (Olympic Charter 2015). The Olympic Charter states that all International Federations are obligated to "encourage and support measures protecting the health of athletes, and encourage and support the development of sport for all". The Olympic Movement Medical Code also regulates that medical staff who work with athletes must ensure athlete safety and well-being by stating, "The Olympic Movement, in accomplishing its mission, should encourage all stakeholders to take measures to ensure that sport is practiced without danger to the health of the athletes ... it encourages those measures necessary to protect the health of participants and to minimize the risks of physical injury and psychological harm" (Olympic Movement Medical Code 2016). Although not all injury surveillance research is associated with the International Olympic Committee (IOC), these statements clearly outline the importance of information attained from this research.

In order to follow these guidelines and ensure the protection of athletes, the van Mechelen model of injury prevention was created and is still followed today (Figure 1) (van Mechelen et al. 1992). The end goal of this theoretical framework is to identify prevention measures to avoid sport-specific injuries. However, in order to accomplish this, there are a few steps to be completed first. The first step requires the collection of information regarding the incidence and severities of injuries in different sports. The first step is essential to identifying a results-based approach of injury prevention because without it, 
the second step that determines mechanism of injuries cannot take place. Preventative measures in step three can then be examined by implementing the same injury surveillance protocol as originally applied in the first step of this model (van Mechelen et al. 1992). If this model is followed and there is an improvement in regards to athlete health (step four), the prevention measures are proven effective for that specific sport or situation.

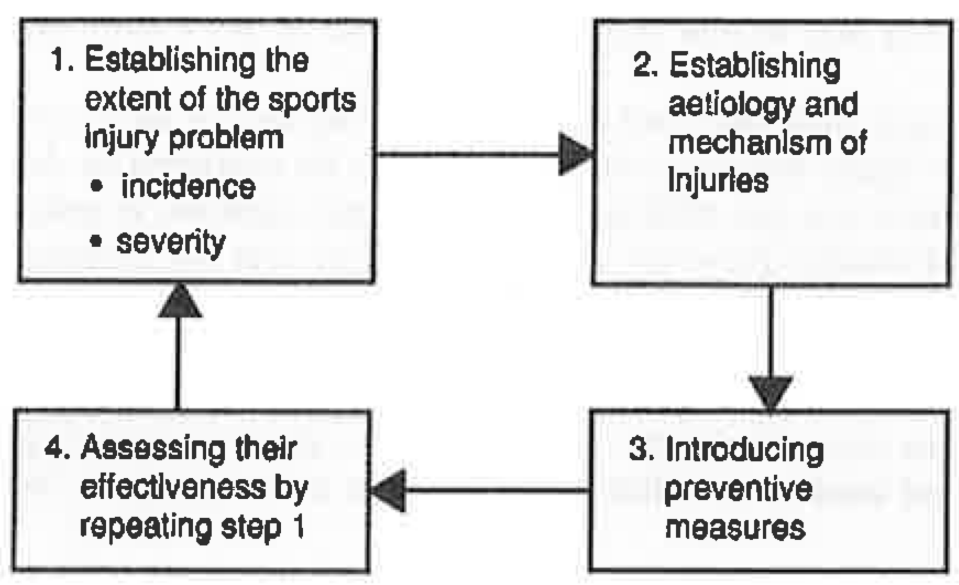

Figure 1: van Mechelen's model of injury prevention (van Mechelen et al. 1992).

Injury and illness surveillance protocols are being implemented in many different sporting-events as the first step to providing prospective and retrospective data regarding athlete health. Injury and illness epidemiology has been considered at the high school level (Hinton et al. 2005, Comstock et al. 2006, Shankar et al. 2007, Borowski et al. 2008, Collins and Comstock 2008, Rechel et al. 2008b, Dompier et al. 2015), the National Collegiate Athletic Association (NCAA) level (Dick et al. 2007a, Kerr et al. 2014), the professional level (Starkey 2000, Fuller et al. 2006, King et al. 2009, Morgan et al. 2010, Elliott et al. 2011, Posner et al. 2011, Jackson et al. 2013, Lynch et al. 2013, Tuominen et al. 2015, Podlog et al. 2015, Chambers et al. 2016, Pollack et al. 2016), the Fédération internationale de natation (FINA) World Championship level (Mountjoy et al. 2010, 2015, Prien et al. 2016), and the IOC level (Junge 2006, Junge et al. 2009, Engebretsen et al. 2010, 2013, Soligard et al. 
2015). Several other International Federations like the International Association of Athletics Federations (IAAF) (Feddermann-Demont et al. 2014), Fédération Internationale de Football Association (FIFA) (Fuller et al. 2006, Junge and Dvorak 2013, Junge et al. 2015), World Rugby

(WR) (Fuller et al. 2015), International Handball Federation (IHF) (Bere et al. 2015a), Federation International de Volleyball (FIVB) (Bere et al. 2015b) have also published injury surveillance data. However, other than the limited research that examined injuries of athletes competing in Canadian Interuniversity Sport (CIS) men's basketball (Meeuwisse et al. 2003), men's football (Meeuwisse et al. 2000), and women's hockey (Schick and Meeuwisse 2003), injury and illness surveillance research has yet to be applied to Canadian sports at the university level (Meeuwisse and Love 1998).

\section{Injury and illness surveillance protocols in elite sport}

Research involving injury and illness surveillance of athletes has received increasing attention by sport medicine researchers to protect athletes and assist in identifying future preventative measures. By doing this, the objective is to protect athletes from these injuries and illnesses, and the negative effect they have on their training, competition, and most importantly for their overall health.

\section{Injury surveillance in high school sports}

In Canada there are very few publications considering the longitudinal injury surveillance of high school student athletes. Although many teams and schools have ways of collecting injury data from athletes, there is still no universal system for collecting injury data on high school athletes in Canada. Some information has been collected by a system called the Canadian Hospitals Injury Reporting and Prevention Program (Pickett et al. 2000). This system was developed to collect injury data from high school-aged children 
with injuries that is based on hospital visits (Pickett et al. 2000). Other individual studies have completed injury surveillance on athletes of this age in sports like ice hockey (Emery and Meeuwisse 2006) and soccer (Emery et al. 2005), but most modify other injury surveillance systems as required.

Although there is a lack of research regarding injury surveillance specific to Canadian high school student athletes, there are many injury surveillance studies and systems that have taken place in high schools in the United States of America (USA). One of the most popular injury surveillance systems is called the High School Reporting Information Online (RIO) and it is used in high schools all across the USA for sports such as baseball (Collins and Comstock 2008), basketball (Borowski et al. 2008), football (Shankar et al. 2007), lacrosse (Hinton et al. 2005), and wrestling (Rechel et al. 2008a), as well as softball and volleyball (Comstock et al. 2006). This weekly electronic surveillance system requires the team trainer or Certified Athletic Therapists/Trainer (CAT) to record any injuries that occur due to participation of high school sport that required medical attention or caused the athlete to miss time from training or competition. Exposure is also recorded by the trainer or CAT and imputed in to the online system (Comstock et al. 2006).

Another surveillance program that researchers have used in the high school setting is the National Athletic Treatment, Injury and Outcomes Network (NATION) (Dompier et al. 2015). This electronic injury surveillance program required team trainers or CATs to record injuries of athletes from 147 high schools in 27 sports in the USA. NATION not only records time loss injuries, but also records non-time loss injuries. Exposure rates were also recorded in this project. Due to the specific injury tracking software, all data could be stripped of identifiers once data was imputed. 


\section{Injury surveillance at the collegiate and university level}

Unlike the NCAA colleges in the USA, University Sports in Canada (U Sports formerly known as CIS) doesn't have a regulated system for injury surveillance. However, there has been limited surveillance of sport-related injuries in U sports in Western Canada for sports like men's basketball (Meeuwisse et al. 2003), men's football (Meeuwisse et al. 2000), and women's ice hockey (Schick and Meeuwisse 2003). The system that this group of researchers used for injury surveillance is called the Canadian Intercollegiate Sport Injury Registry (CISIR). It involved preseason baseline screening of athletes, daily collection of participation for exposure rates, and recording of all individual injury data forms (Meeuwisse and Love 1998). However, this method only includes time-lost injuries and doesn't appear to be actively used since the last publication in 2003.

The NCAA has almost 500,000 student athletes that compete in the 24 sports across their 3 divisions Nationwide ("What is the NCAA," 2015). Early in the $20^{\text {th }}$ century, many NCAA football athletes suffered severe injuries or death from participating in the sport (Dick et al. 2007a; Kerr et al. 2014). The NCAA has taken many steps to ensure the safety of their athletes by collecting injury-related data in sport to provide rule changes and prevention measures for athletes. The NCAA and the National Athletic Therapists Association (NATA) developed their partnership starting in 1982 that allowed certified athletic therapists (CAT) to collect injury data on their athletes during sport. This injury database has now grown into the largest database of sport-related injuries of collegiate athletes in the world (Dick et al. 2007 a; Kerr et al. 2014). Because this data is so valuable for researchers, the NCAA partnered with the Datalys Center for Sports Injury Research 
and Prevention Inc., so that external researchers can requests data sets of 5-year periods of this ISS information for their own research (Kerr et al. 2014).

In a review of the NCAA ISS methods by Kerr et al. (2014), the changes of the injury surveillance since it began in 1982 are discussed. From 1982 to 2004, the NCAA ISS used paper forms that were filled out by the team's CAT as the recording method. The forms included injury and exposure information. At this time, only injuries were recorded if there was training or competition time lost. Data was then mailed or faxed so that it could be input in to the database. From 2004 to 2009, the NCAA provided participating CAT's with electronic software to record injury data. Although the data still had to be input manually, identifiers could now be avoided and data did not have to be mailed. From 2009 to 2014, data was still collected electronically by the team's CAT. However, athlete injuries that did not cause training or competition time loss were also recorded. The electronic system was updated to also identify possible invalid data (Kerr et al. 2014).

\section{Injury surveillance in professional sport}

Due to the context of secrecy in the professional sport environment, the sharing of aggregate health data across competing teams is not practiced. Professional Leagues such as FIFA (Fuller et al. 2006, Junge and Dvorak 2013, Junge et al. 2015), Major League Baseball (MLB) (Posner et al. 2011, Pollack et al. 2016), National Basketball Association (NBA) (Starkey 2000, Jackson et al. 2013, Podlog et al. 2015), National Football League (NFL) (Morgan et al. 2010, Elliott et al. 2011, Lynch et al. 2013, Chambers et al. 2016), National Hockey League (NHL) (Tuominen et al. 2015), Rugby League (King et al. 2009), and Rugby Union (Fuller et al. 2007, Moore et al. 2015) all have some form of injury surveillance system to advance research in injury epidemiology and prevention. 
After a meeting with the FIFA Medical Assessment and Research Centre in 2005, Fuller and colleagues (2006), published a paper regarding a consensus definition of "injury" and explanation of their injury surveillance system. This prospective system uses both paper and electronic recording of injuries, depending on the preference of team trainer or physician. The trainer or physician is required to record assessments of injury location, type, cause, and severity. It also specifies whether an injured athlete requires medical attention, or time missed from training or competition. Additionally, the FIFA system also takes exposure into account throughout athlete injury surveillance (Fuller et al. 2006).

Injury surveillance in the MLB originally began when Posner and Colleagues (2011), analyzed data from the MLB's disabled list. However, this retrospective injury surveillance was based on a public internet website that recorded injuries if a player was going to miss a minimum of 15 days due to the injury (Posner et al. 2011). Although this allowed researchers to determine location, type, and causes of injuries, it lacked the ability to capture athlete's injuries that were less severe than missing 15 days (Pollack et al. 2016). In 2010, the MLB decided to implement a new electronic medical record (EMR) injury surveillance system called the Health and Injury Tracking System (HITS) where the team CAT would input all injury and medical treatment information across minor and major league baseball, regardless if the athlete missed time so that the data could be analyzed for more accurate prevention measures (Pollack et al. 2016).

In the NBA, the CATs for each team preserve a league wide data base to record injuries sustained by athletes and several published studies have been based on this data (Starkey 2000, Jackson et al. 2013, Podlog et al. 2015). Injuries were reported monthly to 
the database electronically by the team's CAT if an athlete missed any training or competition time, received treatment from a physician, or needed emergency care. The information reported within the injury surveillance system was injury location, type, cause, and any time missed (Starkey 2000, Jackson et al. 2013, Podlog et al. 2015).

Many researchers have been able to analyze injury data from the NFL Injury Surveillance System (NFLISS) for further investigation of injury location, type, cause, and mechanisms of specific injuries in hopes of creating rehabilitation and prevention measures for athletes (Morgan et al. 2010, Elliott et al. 2011, Lynch et al. 2013, Chambers et al. 2016). The NFLISS is an electronic injury surveillance registry where team CATs input information if an injury requires an athlete to leave a practice or a game. Although it is possible that injuries may go undetected if players return to the game or practice, the system records information like diagnosis and treatment if the athlete is considered injured (Morgan et al. 2010, Elliott et al. 2011, Lynch et al. 2013, Chambers et al. 2016).

In 2014, McKay and colleagues published a report on the epidemiology of injuries in the NHL from 2006 to 2012. This injury surveillance was conducted using the Athlete Health Management System (AHMS) and required team CATs and physicians to document all injuries electronically. All injury location, type, and cause were documented regardless of time lost. Any missed training or competition time was also recorded (McKay et al. 2014).

There are some differences in opinion when it comes to definitions of an "injury" in Rugby League (King et al. 2009). Nonetheless, there is some injury surveillance that has taken place and the surveillance system used for this league is only based on paper forms that are completed by the team trainers. The information collected includes athlete 
information, injury location, type, cause, and the length of injury in days (King et al. 2009). Unlike Rugby League, Rugby Union have developed a more sound definition of an "injury" (Fuller et al. 2007, Moore et al. 2015). Moore and colleagues (2015) implemented injury surveillance on a single Rugby Union men's international team from 2011 to 2014. In this study, injuries were recorded if there was any physical complaint that required an athlete to miss training or competition time. Exposure rates were also recorded for this research (Moore et al. 2015).

\section{Injury and illness surveillance during FINA World Championships \& Olympic Games}

Before 2009, there was no measure of illness incidence rates in elite aquatic multisport events (Mountjoy et al. 2010). In 2009 at the $13^{\text {th }}$ FINA World Championships, FINA decided to implement an illness surveillance protocol in addition to their already implemented injury surveillance protocol (Mountjoy et al. 2010). During the competition, team physicians and physiotherapists were asked to fill out forms for every injury or illness suffered by their athlete regardless of whether the injury or illness caused them to miss training or competition time. Part of this project was to also test the feasibility of this protocol at a multi-sport event in hopes of applying it at the 2010 Olympic Winter Games in Vancouver (Mountjoy et al. 2010). Similar projects were conducted and data are compared by FINA at the $15^{\text {th }}$ and $16^{\text {th }}$ FINA World Championships in 2013 and 2015, respectively (Mountjoy et al. 2014, Prien et al. 2016).

Prospective injury surveillance research has been conducted throughout several Olympic Games (Junge 2006, Junge et al. 2009, Engebretsen et al. 2010, 2013, Soligard et al. 2015). At the 2008 Olympics Summer Games in Beijing, the group of researchers developed an athlete injury surveillance system for multi-sport events by reviewing methods of 
previous injury reporting systems (Junge et al. 2009b). This system included definitions of injuries, instructions of how physicians should record information, and reporting sheets for all injuries to be recorded.

In 2010 at the Olympic Winter Games in Vancouver, the members of the IOC extended their athlete surveillance research by including athlete illnesses in addition to athlete injuries into the surveillance system (Engebretsen et al. 2010). The methodology of this reporting system was based on the injury surveillance at the 2008 Olympic Summer Games (Junge et al. 2009) and the 2009 FINA World Championship (Mountjoy et al. 2010). This system used daily injury and illness forms and a database where all athletes were assigned a specific accreditation number. This methodology was continued during the London 2012 Olympic Summer Games (Engebretsen et al. 2013) and the 2014 Olympic Winter Games (Soligard et al. 2015) to assess athletes' health on a holistic level and to provide insight into future prevention measures (Junge et al. 2008b).

\section{Epidemiology of injuries and illnesses in sports}

The first step of van Mechelen's model of injury prevention is to measure the incidence and severity of athlete injuries in different sports (van Mechelen et al. 1992). In order to progress to the next step of the injury prevention model, we must first understand the epidemiology of sport-specific injuries. For the purpose of this thesis, this section of the literature review examines the epidemiology of sport-specific injuries and illnesses at the CIS and NCAA level of sport. If there is no information available for a sport at this level, information is provided from either the high school or professional level of sport. The sports and level of sport reviewed are specific to the sports that are included in this thesis as part of the injury and illness surveillance of varsity athletes at the University of Guelph. 


\section{Men's baseball}

In a 16-year review of NCAA men's baseball injuries that was completed by Dick and colleagues (2007f), it was found that men's baseball had a rather low injury rate compared to other NCAA sports. They also found that injury incidence was three times greater in games (5.78 injuries/1000 games) than in practices (1.85 injuries/1000 practices), and that $25 \%$ of all injuries were considered severe (at least 10 days missed due to injury). In regard to injuries sustained in practice, roughly $2 / 3$ of injuries were non-contact injuries. However, $45 \%$ of game injuries occurred in contact mechanisms with either the ball, the wall, or the ground, and $42 \%$ of injuries occurred through non-contact mechanisms. In practice, the most common injuries were shoulder muscle or tendon strains (10\%), ankle ligament sprains $(8.5 \%)$, upper leg muscle or tendon strains $(8.3 \%)$, shoulder tendinitis (6.7\%), and lower back muscle or tendon strains (4.2\%). Comparably, the most common injuries in a game were upper leg muscle or tendon strains (11\%), ankle ligament sprains (7.4\%), shoulder muscle or tendon strains (6.5\%), knee internal derangement injuries (3.7\%), concussions (3.3\%), and elbow ligament sprains (3.2\%) (Dick et al. 2007f).

\section{Men's and women's cross country}

In a systematic review of lower extremity running injuries in long distance runners by van Gent and colleague (2007), the incidence of lower extremity running injuries ranged from 19.4 to $79.3 \%$ depending on the study, and the most common location of injury was the knee. Because of this large gap in incidence rate, it is clear that more standardized research needs to be done to ensure accuracy of measuring this injury data (van Gent et al. 2007). However, in 2016 there was a 5-year review of the NCAA men's and women's crosscountry injuries that provided a good summary of their findings in regards to injury 
surveillance in the sport (Kerr et al. 2016). This study found that the majority of injuries that occurred were lower extremity overuse injuries and there were 1.25 times more injuries in women's cross-country running than in men's cross-country running. Thompson and colleagues (2004), also outlined similar findings for female collegiate cross-country runners.

Female cross-country runners' injury incidence was found to be 5.85 injuries/1000 $\mathrm{AE}$ (athlete exposures) with the majority of their injuries occurring from overuse (53.3\%). The majority of women's injuries occurred in training with $88.8 \%$ of their injuries being sustained in practice and only $11.2 \%$ of their injuries occurring in competition. However, injury rates for training and competition were not different when athlete exposure was accounted for. Time loss incidence rates were 3.44 injuries/1000 AE and non-time loss injury incidences were 2.41 injuries/1000 AE. The most common injuries sustained by female cross-country runners were thigh strains $(6.5 \%)$, lower leg inflammation $(6.5 \%)$, lower leg tendonitis (6.2\%), lower leg strains (5.8\%), and foot inflammation (5.8\%) (Kerr et al. 2016).

Male cross-country runners injury incidence was found to be 4.66 injuries/1000 AE with the majority of their injuries also occurring from overuse (57.6\%). Similar to women's cross country runners, injury rates (per $1000 \mathrm{AE}$ ) did not differ between training and competition even though the majority of men's injuries occurred in training $(92.1 \%$ in training and 7.9\% in competition). Time loss incidence rates were 2.85 injuries/1000 AE and non-time loss injury incidences were 1.85 injuries/1000 AE in these male crosscountry runners. The most common injuries sustained by male cross-country runners were 
ankle sprains (11.1\%), lower leg inflammation (9.7\%), lower leg tendonitis (8.3\%), lower leg strains (6.9\%), and foot inflammation (6.9\%) (Kerr et al. 2016).

\section{Women's field hockey}

Due to the limited literature available to review the epidemiology of injuries in women's field hockey at the university or collegiate level, a 15-year review of NCAA female field hockey injuries is likely the best representation and comparison of this data (Dick et al. 2007b). This review outlines the findings that injury incidences were twice as high in games (7.87 injuries/1000 AE) than in practices (3.7 injuries/1000 AE). Lower extremity injuries accounted for $40 \%$ of game injuries and $60 \%$ of practice injuries. In reference to injury severity, $\sim 15 \%$ of injuries sustained in a game and $\sim 13 \%$ of injuries sustained in a practice caused the athlete to miss at least ten days of training or competition. Injuries sustained in a game were mostly caused by "other contact" (contact with the ball, sticks, or the field) (59.8\%), with contact with the ball being the greatest cause of injury (29.2\%), and majority of injuries sustained in practice were non-contact injuries (64\%). The most common injuries that female field hockey players sustained were upper leg muscle or tendon strains $(26.9 \%)$, ankle ligament sprains (15\%), hip or pelvis muscle or tendon strains $(9.9 \%)$, knee internal derangement $(7.8 \%)$, and lower back muscle or tendon strains (5.2\%). Similarly, the most common injuries sustained in games were ankle ligament sprains (13.7\%), knee internal derangement (10.2\%), concussions (9.4\%), upper leg muscle or tendon strains (7.0\%), and finger fractures (6.5\%). Another study also found that NCAA women's field hockey players had one of the highest rates of overuse injuries (70.5 overuse injuries/1000 AE) in comparison with other sports (Yang et al. 2012). 


\section{Men's football}

Although much of the injury surveillance for football athletes is conducted in the USA, Meeuwisse and colleagues (2000), completed a 5-year overview of CIS football in Western Canadian universities. While this research is not the most recent injury surveillance of football injuries, it is likely still the best comparison to football athlete injuries at the University of Guelph given that the leagues follow the same rules and CIS teams from the East and West meet each year in the National championship (Meeuwisse et

al. 2000). Over the 5-year study, injury proportion ranged from 53.5 to $60.4 \%$, and increased each year from 1993 to 1997 (ranging from 303 to 399 injuries per year). Of all injuries that occurred in football athletes, $65 \%$ of injuries were related to contact between two players. The most common injuries sustained were concussions (5.6\%), hamstring strains (4.5\%), brachial plexus injuries (4.3\%), quadriceps contusions (3.8\%), and knee MCL sprains (3.5\%). The average time lost per injury was 5.82 training or competition sessions, with forearm (14.6), knee (13.5), leg (6.5), shoulder (6.3), and ankle (6.2) injuries causing athletes to miss the most sessions of training or competition.

\section{Men's and women's golf}

In the sport of golf, there is no injury surveillance research in university or collegiate athletes, and very little research at other competitive levels in the literature. Any golf injury data reported in men and women is usually collected retrospectively through surveys or questionnaires that are submitted by amateur and professional golfers at different golf courses (Batt 1992, Gosheger et al. 2003). One researcher, in 1992, provided 461 golfers with an injury survey that asked about past and current injuries (Batt 1992). Of the 193 golfers who responded, $57 \%$ of them reported at least one injury ( $56 \%$ of men and 
$59 \%$ of women). Although not all injuries were diagnosed, the most common complaints from the men involved their wrist (28\%), back (25\%), elbow (8\%), and knee (8\%), while women's most common complaints involved their elbow (50\%), shoulder (25\%), and wrist (12\%). The golfers also reported that the two most common injuries were believed to be overuse and incorrect swings (Batt 1992).

Another study by Gosheger and colleagues (2003), randomly interviewed 703 golfers (510 men and 193 women) over two seasons that were either professionals or amateurs. Of all of the injuries reported, $82.6 \%$ were considered to be overuse injuries and $17.4 \%$ were considered to be traumatic events. Both professional and amateur golfers reported a very high injury rate that was greater than one injury per golfer over the two seasons (3.06 injuries per professional golfer and 2.07 injuries per amateur golfer). Professional golfers' most common injuries were sustained in their back (34.5\%), wrist (20\%), and shoulder (12.7\%), while amateur golfers' most common injuries were sustained in the elbow (24.9\%), back (24.7\%) and shoulders (18.6\%). Injuries to the thoracic spine cause the most time missed by golfers (137 days), and there were no statistical differences between men and women (Gosheger et al. 2003).

\section{Men's and women's lacrosse}

One study of injury epidemiology at the university or collegiate level for men's and women's lacrosse is outlined in two 16-year reviews of injury surveillance in NCAA lacrosse athletes (Dick et al. 2007c, e). Injury rates for men's lacrosse were 12.6 injuries/1000 AE for games and 3.24 injuries/1000 AE for practices (Dick et al. 2007e). Women's lacrosse injury rates were also twice as high in games compared to practice (7.15 injuries/1000 AE for games vs. 3.30 injuries/1000 AE for practices) (Dick et al. 2007c). 
Approximately 53 and $60 \%$ of those injuries were reported as lower extremity injuries for men and women lacrosse athletes, respectively.

The most common injuries for men were ankle ligament sprains (11.3\%), knee internal derangements (9.1\%), concussions (8.6\%), and upper leg contusions $(8.0 \%)$ in games, while most common practice injuries were ankle ligament strains (16.4\%), upper leg muscle strains (11.4\%), and knee internal derangements (7.1\%). The majority of game injuries (50\%) occurred due to player contact, but most of practice injuries (45.9\%) were non-contact injuries (Dick et al. 2007e). Similarly, the most common injuries for women were ankle ligament sprains (22.6\%), knee internal derangements (14\%\%), concussions $(9.8 \%)$, and upper leg muscle strains $(7.2 \%)$ in games, while most common practice injuries were ankle ligament strains (15.5\%), upper leg muscle strains (11.7\%), and knee internal derangements (6.1\%). Most of these injuries were considered non-contact injuries (44.3\% of game injuries and 62\% of practice injuries) (Dick et al. 2007c). In both men's and women's lacrosse, $\sim 21 \%$ of injuries caused the athlete to miss at least 10 days of training or competition (Dick et al. 2007c, d).

\section{Men's and women's rowing}

Although there is no injury surveillance research published at the collegiate level for rowing athletes, one study that looked at acute and overuse injuries in NCAA athletes found that out of 16 sports, women's rowing had the most overuse injuries (Yang et al. 2012). Of the 98 injuries over the three seasons, 56 injuries were considered overuse injuries and 42 were acute injuries. Several other studies have also reported a low injury rate for the sport of rowing (Smoljanovic et al. 2011, Yang et al. 2012, Engebretsen et al. 2013). At the 2012 Olympic Summer Games, it was found that $4.1 \%$ of female rowers and $2.8 \%$ of male rowers 
reported an injury (Engebretsen et al. 2013). In regard to mechanism of injury, it was found that $73.8 \%$ of injuries were considered overuse and $26.2 \%$ were considered traumatic injuries in junior elite rowers (Smoljanovic et al. 2011). This research also showed that the most common injuries were located in the athletes' lower back (female: 29.9\%, male: 34.4\%), knee (female: 17.9\%, male: $19.6 \%$ ), and wrist or forearm (female: $13.6 \%$, male: 9.6\%). Some of these rowers sustained 2 or more injuries throughout these seasons but only $3.1 \%$ of these injuries were considered to be of major severity ( $>1$ month missed) (Smoljanovic et al. 2011).

\section{Men's rugby}

Although Rugby is a popular sport in many parts of the world, there is very little research published in North America on men's rugby injury surveillance patterns. However, there is one paper that conducted injury surveillance in men's rugby union in the New England region (Kerr et al. 2008). This group of researchers found that injury rates for men's union rugby in this area were 5.5 injuries/1000 AE in practices and 22.5 injuries/100 AE in games. While $56 \%$ of practice injuries were considered minor $(<7$ days of training or competition missed), an alarming amount of game injuries (56\%) were considered severe and caused the athlete to miss at least 7 days of training or competition. The most common injury locations in practice and games were the head or face $20.9 \%$ of practice injuries, $27.2 \%$ of game injuries), shoulder $(12.7 \%$ of practice injuries, $12.8 \%$ of game injuries), knee (11.2\% of practice injuries, $12.8 \%$ of game injuries), and ankle $(11.2 \%$ of practice injuries, $11.2 \%$ of game injuries). In addition to this, sprains ( $25.4 \%$ of practice injuries, $21.7 \%$ of game injuries), strains $(23.1 \%$ of practice injuries, $12.8 \%$ of game injuries), and concussions (6.7\% of practice injuries, $12.8 \%$ of game injuries) were the 
most common types of injuries for these athletes in both practices and games. Almost half (48\%) of the game injuries were caused by tackles (Kerr et al. 2008).

\section{Men's and women's soccer}

In examining the literature of men's and women's soccer, concussion rates have been shown to be amongst the highest in men's soccer (0.49 injuries/1000 AE) and women's soccer (0.63 injuries/1000 AE) in comparison to other sports like baseball, basketball, football, and wrestling (Gessel et al. 2007). There are two 15-year reviews that outline the epidemiology of injuries that are associated with soccer (Agel et al. 2007, Dick et al. 2007d). Women's injury rates were found to be 3 times as high in games (16.4 injuries/1000 AE) compared to practice (5.23 injuries/1000 AE) (Dick et al. 2007d). Of these injuries, $70 \%$ were reported to be lower extremity injuries. Player contact (54\% of injuries) was the main cause of the game injuries, while the main cause of practice injuries were considered non-contact ( $56 \%$ of injuries). The most common injuries sustained by women were ankle ligament sprains (18.3\%), knee internal derangements (15.9\%), concussion (8.6\%), and leg contusions (8.3\%) in games, and upper leg muscle strains (21.3\%), ankle ligament sprains (15.3\%), knee internal derangements $(7.7 \%)$, and pelvis or hip muscle strains (7.6\%) during practice. Based on the severity of these injuries, $22 \%$ of game injuries and $17 \%$ of practice injuries required at least 10 days of time loss from training or competition (Dick et al. 2007d). Another study in NCAA athletes found that women's soccer athletes had some of the highest acute (19 injuries/1000 AE) and overuse (4.8 injuries/1000 AE) injury rates compared to other collegiate sports (Yang et al. 2012).

The injury rates for men were also found to be more than 3 times the amount in games (18.8 injuries/1000 AE) versus practice (4.34 injuries/1000 AE) (Agel et al. 2007). 
More than two thirds of these injuries were considered lower extremity injuries, while $61 \%$ of game injuries were caused by player contact and $47 \%$ of practice injuries were considered non-contact injuries. The most common injuries found in these male soccer players were ankle ligament sprains (17\%), internal knee derangement (11\%), upper leg

muscle strains (8.2\%), and upper leg contusions (6.4\%) in games, and ankle ligament sprains (17.4\%), upper leg muscle strains (16.6\%), pelvis or hip muscle strains (7.8\%), and knee internal derangements (7.7\%). Based on the severity of these injuries, $18.7 \%$ of game injuries and $14.6 \%$ of practice injuries required at least 10 days of time loss from training or competition (Agel et al. 2007).

\section{Feasibility and implementation of injury and illness surveillance protocols}

Regardless of whether injury and illness data are published for research, creating accurate injury and illness surveillance methods ensures that the medical staff is acquiring the correct information about their athletes. Implementing a feasible and high-quality injury and illness surveillance system requires clear and consistent definitions of injury and illness, the collection of the appropriate injury and illness information through applicable collection methods, and the risk of injury though exposure due to training and competition (Finch 1997, Junge and Dvorak 2000, Brooks and Fuller 2006). Implementing the protocol must have a balance between the ability of simple reporting, acceptability, timely recording, and feasibility (Meeuwisse and Love 1997).

"Injury" and "illness" definitions in FINA and IOC prospective athlete surveillance

Without clear and consistent definitions, information cannot be collected or compared in a uniform manner across multiple studies (Junge et al. 2008b, Ljungqvist et al. 2009, Mountjoy et al. 2015, Prien et al. 2016). This also means that data before and after 
implementation of prevention measures cannot be compared. The current definition of "injury" is, "a physical complaint or observable damage to body tissue produced by the transfer of energy experienced or sustained by an athlete during participation in training or competing in a varsity sport, regardless of whether it received medical attention or its consequences with respect to impairments in competition or training" (Fuller et al. 2007, Timpka et al. 2014a).

In extension of this, it has been suggested that separating injuries into "traumatic injuries" and "overuse injuries" based on their mechanism or causation of injury can provide needed information for any implementation of future prevention strategies (Meeuwisse 1994, Bahr and Krosshaug 2005, Meeuwisse et al. 2007, Timpka et al. 2014b). A "traumatic injury" can also be subdivided into injuries caused by athlete contact, contact with an object, or non-contact, and is defined as, "an injury caused by a single, clearly identifiable energy transfer" (Fuller et al. 2007, Timpka et al. 2014a). Conversely, an "overuse injury" can be classified as an acute or chronic onset of injury and is defined as, "a condition caused by multiple accumulative bouts of energy transfer without a single, identifiable event responsible for the injury" (Fuller et al. 2006, Timpka et al. 2014a).

FINA and the IOC have also decided to use the classification system by Hamilton and colleagues (2011) for other injury definitions (Mountjoy et al. 2015). An index injury is defined as, "the first recorded injury in a series of injuries constituting a recurrent condition". An exacerbation is defined as, "an injury to the same location and of the same type as the index injury, where the index injury has not completely healed". A re-injury is defined as, "injury to the same location and of the same type as the index injury, where the index injury has completely healed". A local injury is defined as, "an injury to the same 
location but a different type from the index injury". A new injury is defined as, "an injury to a different location from the index injury".

In regards to athlete surveillance research, an "illness" is defined as, "a complaint or disorder experienced by an athlete that is not related to injury, regardless of whether it received medical attention or its consequences with respect to impairments in training or competing in a varsity sport" (Timpka et al. 2014a). An illness can be defined as either physical trauma or psychological trauma experienced during sport or suffered outside of sport (Mountjoy et al. 2015).

\section{Compliance and acceptability of injury and illness surveillance}

In regard to compliance and acceptability of injury and illness reporting systems, the IOC approach explains that response rates should be $\geq 80 \%$ to avoid any bias (Junge et al. 2008b). Over the past decade, response rates have continued to grow in a positive direction during FINA World Championships and the Olympic Games (Junge et al. 2008a, Prien et al. 2016). In a review by Prien and colleagues (2016), it outlined the fact the injury and illness rates have continued to grow in FINA World Championships from 2009 to 2015. However, compliance and response rate of report forms returned have also increased, showing that team's medical staff and athletes are becoming more accepting of injury and illness protocols rather than FINA athletes actually becoming injured or ill more often. The number of reporting countries (2009: 42.2\%, 2013: 66.7\%, 2015: 82.3\%), and reporting form response rates (2009: 42.4\%, 2013: 66.7\%, 2015: 82.3\%) has increased in each of the three championships (Mountjoy et al. 2010, Prien et al. 2016).

Similarly in the Olympics Games, there has been an increase in compliance and injury and illness reporting response rates over the last decade. At the 2004 Olympic 
Summer Games in Athens, injury surveillance took place across only 14 sports but the system used provided a response rate of $>90 \%$ (Junge 2006). At the 2008 Olympic Summer Games in Beijing the response rate decreased to $72.1 \%$, however the participation rate in the study increased immensely to 88\% of all registered athletes (Junge et al. 2009). In 2010 at the Olympic Winter Games in Vancouver, illnesses were also recorded among athletes and there was still an increase in response rate to $82 \%$ for return injury and illness report forms (Engebretsen et al. 2010). Recently, an outstanding compliance and response rate of $>93 \%$ was achieved at the 2014 Olympic Winter Games in Sochi (Soligard et al. 2015).

Very similar research was conducted at the 2012 Winter Youth Olympic Games (Ruedl et al. 2012) and the 2012 Paralympic Games (Derman et al. 2013). These two multisport events had excellent compliance and a response rate of $100 \%$. Researchers at the 2012 Winter Youth Olympic Games made note that athletes and training staff were only located in one village during the competition, which made it easier to acquire any missing data (Ruedl et al. 2012). In the study conducted during the 2012 Paralympic Games, the research team used an online web-based injury and illness surveillance system, which they credit to improve the response rate success (Derman et al. 2013).

\section{Methodological considerations for controlled injury and illness surveillance}

As with any research, it is clear that there are limitations that should be taken into consideration when conducting injury and illness surveillance depending on the specific protocol and the cohort being studied. Regardless of compliance of athletes and medical staff, and response rate of forms, there is always the possibility of underreporting because of the increase in workload for team medical staff (Junge et al. 2008b, Ljungqvist et al. 2009, Kerr et al. 2014, Prien et al. 2016). Although obtaining all needed information 
remains the most important task, it is likely that the least time consuming option for the surveillance system will acquire, not only the highest response rate, but also the best chance of accurate reporting of injuries and illnesses. While it is important to incorporate adjustments as technology advances to make this research easier for those recording, measures may be different and the reporting medical staff will have to adapt to the new system, which may affect response rates and reporting accuracy (Kerr et al. 2014).

Secondly, it is essential for injury and illness definitions and methods to be consistent across injury and illness surveillance to ensure that results are reliable and can be compared between different studies (Junge et al. 2008b, Ljungqvist et al. 2009, Mountjoy et al. 2015, Prien et al. 2016). Without definitions being consistent, it would be unclear whether the results were different or if altered methodology caused disparities. For example, using the injury and illness surveillance data from the FINA World Championships and Olympic Games have been unable to quantify training or competition AE data due to the complexity of the multisport tournament (Junge 2006, Junge et al. 2009, Engebretsen et al. 2010, 2013, Soligard et al. 2015). Although these studies can compare incidence rates of competitions and sports between one another, they are unable to compare incidence rates obtained by other studies that included an AE measure. One other example of a limitation is from the 2010 Olympic Winter Games injury and illness surveillance, where injury and illness severity was not clearly reported by some medical staff and athletes (Engebretsen et al. 2010). This outlines the importance of all measures because when even one parameter is not reported, it impedes direct comparisons with other studies. 
Finally, very limited research in this field has tested the accuracy and validity of the methods and findings of injury and illness surveillance due to the fact that the majority of research using this model to assess athletes is at multisport tournaments (Junge 2006, Junge et al. 2009, Engebretsen et al. 2010, Mountjoy et al. 2010, Derman et al. 2013, Soligard et al. 2015, Prien et al. 2016). This was assessed in the NCAA with men's and women's soccer data from 2005 to 2007, and the research found that the NCAA ISS captured $88.3 \%$ of injuries (Kerr et al. 2014). Although this may be a lengthy task, it could be very important for estimating the accuracy and validity of this research so that proper modifications can be made to athlete surveillance systems in the future.

\section{Conclusion}

With the ultimate goal of keeping athletes healthy, it is important for the medical staff to conduct, or at least participate in, injury and illness surveillance research. The model of injury prevention developed by William van Mechelen's outlines that until athlete surveillance is carried out, it is impossible to directly understand the mechanism of injury and what prevention measures might be most effective for maintaining athlete health (van Mechelen et al. 1992). Implementing injury and illness surveillance in multisport events allows for sport-specific injury prevention, which can range from modification of equipment, rules and regulations in sport, sport-specific training methods, training volume, pre-participation screening methods, and education to athletes.

One of the most important findings of this literature review is that although there is a massive amount of data available regarding athlete injury surveillance in high schools in the USA, the NCAA, professional sports, the Olympic Games, and various International Federations, there is very limited data pertaining to the Canadian university level of sport. 
Not only is there limited data, much of the existing data at this competition level is NCAA data that is more than 10 -years old. In addition to this, there is still no standard of injury and illness surveillance of athletes at the Canadian university level in the scientific literature. This is concerning due to the fact that changes in sport over the past decade might result in different findings, and that there are over 9,000 student athletes that participate in university sport in Ontario alone (OUA 2014-2020 Strategic Plan). In the future, it will be important to solidify injury and illness surveillance in athletes at all levels, to record training injury and illness surveillance rather than only during competition, to improve health education to all athletes and medical staff, and identify sport-specific prevention measures to optimize overall health of athletes (Prien et al. 2016). 


\section{CHAPTER 2: AIMS OF THE THESIS}

\section{Problem}

A considerable amount of the contemporary research in sport involves trying to improve athletes' performance through ergogenic aids and different training protocols. However, the maintenance of overall health of the athlete is sometimes overlooked when considering performance and their ability to compete. To have success in sport, it is imperative for coaches and medical staff (Finch et al. 1999, Olympic Charter, 2015, Olympic Movement Medical Code, 2016) of the team to understand importance of athlete health throughout training and competition seasons. The health of an athlete is essential for their best performance, and until researchers have sufficient sport-specific injury and illness information, preventative measures cannot be tested or implemented to improve overall health and performance.

Over the past decade, much effort has been made to secure a reliable and valid model for injury and illness surveillance at many levels of sport, to better understand risks and occurrences within individual sports. Several protocols have been tested at many levels of sport including NCAA, professional, and on the world stage. However, to our knowledge there have been no attempts to implement these protocols in Canadian university sport. By testing and implementing a continuous injury and illness surveillance protocol with varsity athletes in Canada, such as the one recommended by FINA, it would provide 1) detailed information about the health risks of specific sports on athletes, 2) their recovery following injuries and illnesses, and 3) identify plausible preventative measures for future intervention. 


\section{Purpose}

The purpose of this research study was to implement and assess an injury and illness surveillance system for varsity athletes from 17 different sports at the University of Guelph. Another aim of this study was to identify injury and illness characteristics and patterns in various varsity sports at the University of Guelph. The outcome measures from the implementation of this surveillance protocol included: the measurement of the compliance and feasibility of the protocol, the time of season the injury and illness occurred, injury location, illness diagnosis, injury or illness cause, the team trainer's and athlete's perceived severity of the injury or illness, injury or illness treatment, the athlete's exposure to training and competition during recovery, any missed or limited training and/or competition during recovery, and total recovery time. All of this information will be used in future research to develop and assess sport-specific pre-season screening and preventative measures for varsity athletes.

\section{Hypotheses}

1) It was hypothesized that implementing this new injury and illness surveillance system would provide a successful compliance rate of at least $80 \%$.

2) It was expected that the majority of athlete injuries would involve the lower extremities.

3) It was expected that gender differences in illness and injury patterns would emerge.

4) It was expected that contact varsity sports would have higher injury rates and more severe injuries than non-contact varsity sports. 
5) Lastly, it was expected that more injuries would occur in competition compared to training. 


\section{CHAPTER 3: METHODS}

This study used a prospective, longitudinal, and observational design. The cohort of team trainers and athletes from all 17 varsity sports at the University of Guelph were asked to participate in this research that involved the collection of their injury and illness information during the course of their competitive season. All injuries and illnesses of the athletes were recorded from the time of their first competition at the start of their season until their competitive season ended. The injury and illness surveillance protocol was not implemented during the preseason as team rosters were not uniformly identified across all sports until the first competitive event in the season. For the purpose of this thesis, only the data from the Fall 2016 semester sports (14 varsity teams) will be presented. The women's rugby team is included in the research for the compliance section of the study, however the majority of the team chose not to participate in the study and therefore no data were collected. The men's rugby "seconds team" (players that were not starters) participated in exhibition games outside of Ontario University Association (OUA) competition, therefore only the athletes that were starters on the men's rugby team were included in the study. The length of time of collection ranged from 6 to 12 weeks depending on the specific sport.

\section{Subjects}

Twenty-nine team trainers from 8 varsity sports (14 teams) provided injury and illness information on 338 varsity athletes (99 females and 239 males) from the University of Guelph during the Fall 2016 semester and competition season. The varsity athletes' mean $( \pm$ SD) age, height, and weight were $20.1 \pm 1.8$ years, $1.8 \pm 0.1 \mathrm{~m}, 80.5 \pm 19.2 \mathrm{~kg}$. All subjects were informed of the experimental procedure and any associated risks before 
written consent to participate in the experiment was obtained. The Research Ethics Board of the University of Guelph provided ethical approval for this research.

\section{Study Design}

\section{Defining injury and illness, and team trainers}

For the purpose of this study, injuries and illnesses were recorded as either new or recurrent after return to full participation in sport. Any injuries that occurred before the start of the competition season, or outside of the sports' training or competition, were not recorded. It was instructed that all illnesses were to be recorded whether they were related to the sport or not. Injury and illness severity was defined as "mild" if they missed $\leq 7$ days, "moderate" if they missed 8-28 days, and severe if they missed $\geq 28$ days (Mountjoy et al. 2015).

In an effort to establish clear definitions, an injury was defined as, "a physical complaint or observable damage to body tissue produced by the transfer of energy experienced or sustained by an athlete during participation in training or competing in a varsity sport, regardless of whether it received medical attention or its consequences with respect to impairments in competition or training" (Fuller et al. 2007; Timpka et al. 2014). Similarly, an illness was defined as, "a complaint or disorder experienced by an athlete that is not related to injury, regardless of whether it received medical attention or its consequences with respect to impairments in training or competing in a varsity sport" (Timpka et al. 2014).

\section{Team trainers}

Varsity team trainers consisted of certified athletic therapists, student athletic therapists from Sheridan College (Brampton, ON), and volunteer undergraduate student 
trainers from the University of Guelph. The team trainers were placed with specific teams by the Athletic Therapy Department staff at the University of Guelph. Their placement depended on team size, men's and women's sports, team schedules, and whether the sport is contact or non-contact. Unfortunately, five of the fourteen teams were not assigned a team trainer for the season. In the case of men's and women's cross country, one of the coaches was the acting team trainer so that data could still be collected. Similarly, for men's rowing, women's rowing, and women's lacrosse, a member of the research team was the acting team trainer.

\section{Implementation}

The lead investigator presented the proposed research to any attending varsity coaches during a meeting that was held 6-weeks prior to the fall 2016 semester. During the first week of the semester, another meeting was held by the research team to explain the full injury and illness surveillance protocol to all varsity team trainers. The team trainers were guided through each of the required injury and illness report forms that would be filled out and given the chance to ask any questions. The team trainers were also given a detailed instruction booklet reviewing the study protocol with informational examples for several different injuries and illnesses. Before the beginning of the each team's season, a researcher explained the study to the athletes and asked for consent to allow their injury and illness information to be collected throughout the season by the trainer in order to be used in this research.

\section{Measurements and experimental protocol}

The protocol of this newly implemented injury and illness surveillance study involves the completion of three reporting forms. The "Weekly summary of injuries and 
illnesses reporting form" and the "Athletic therapist/student trainer injury and illness reporting form" were both filled out by the trainer of the varsity team every 7 days until the affected athlete was healthy. The "Athlete injury and illness reporting form" was filled out by the athlete who sustained the injury or illness every 7 days until the injury or illness subsided. Injuries and illnesses from athletes were recorded starting from the first competition of their season, to the final competition of their season or playoffs, based on their respective teams. One of the researchers met with teams every few weeks to answer questions, to encourage compliance, and to collect reporting forms from prior weeks of recording. Our research team also made themselves available through email and phone for further support. Other than the cross country team, all reporting was completed on paper forms. The cross-country data was collected electronically by the team physiologist and then transferred to paper forms by our research team.

\section{Weekly summary of injuries and illnesses reporting form}

A summary of the weekly injuries and illnesses was completed by the team trainer, which provided athlete gender and age, details of the sport, diagnosis of illness, date of the injury or illness, injured body part or affected system by illness, type of injury or main symptoms of illness, cause of injury or illness, and estimated absence in days (Figures 2 and 3).

\begin{tabular}{|l|l|l|l|l|l|l|}
\hline \multicolumn{2}{|l|}{ age } & \multicolumn{2}{l|}{ sport and event } & \multicolumn{2}{l|}{ round / heat or training } & date \& time \\
\hline injured body part, side & code & type of injury & code & cause of injury & code & absence in days \\
\hline
\end{tabular}

Figure 2: Weekly report that team trainers filled out when an athlete was injured. 


\begin{tabular}{|l|l|l|l|l|l|l|}
\hline \multicolumn{2}{|l|}{ age } & \multicolumn{2}{l|}{ sport and event } & \multicolumn{2}{l|}{ diagnosis } & occurred on (date) \\
\hline affected system & code & main symptom(s) & code & cause of illness & code & absence in days \\
\hline
\end{tabular}

Figure 3: Weekly report that team trainers filled out when an athlete was ill.

Definitions and codes were given on the second page of the "Weekly summary of injuries and illnesses report form" (appendix 1). Examples of this completed form are shown in appendix 4.

\section{Athletic therapist/student trainer injury and illness reporting form}

An individual reporting form was filled out for each injury or illness that an athlete sustained. This form included information regarding the athletes training and competition exposure, the trainers' perceived severity, and any treatment in the 7 days following the injury or illness.

\section{Exposure in training (in minutes per day)}

Please indicate day of injury by circling one of the days below.

n no training in past 7 days

\begin{tabular}{|l|l|l|l|l|l|l|l|}
\hline & Monday & Tuesday & Wednesday & Thursday & Friday & Saturday & Sunday \\
\hline Full & & & & & & & \\
\hline Limited & & & & & & & \\
\hline
\end{tabular}

Figure 4: Exposure in training section of team trainers' report.

In each of the 7 days following the injury or illness, the team trainer recorded the athlete's exposure to training in minutes and whether it was full or limited training (Figure 4). The trainer also recorded any exposure the athlete had to competition in the 7 days following the injury or illness (Figure 5). 
Exposure in competition:

$\square$ no competition in past 7 days

Total days present at a competition:

Event competed in:

/ number of starts or matches:

Event competed in: / number of starts or matches:

Figure 5: Exposure in competition section of team trainers' report.

\begin{tabular}{|l|l|}
\hline 1. Has the athlete had any difficulties in participating in & 2. How much did the athlete modify the volume, content \\
normal training and competition due to injury, illness & or intensity of training due to injury, illness or other \\
or other health problems during the past 7 days? & health problems during the past 7 days? \\
$\square \quad$ full participation without health problems & $\square$ minor \\
$\square \quad$ full participation but with injury / illness & $\square$ moderate \\
$\square \quad$ reduced participation due to injury / illness & $\square$ major \\
$\square$ could not participate due to injury / illness & $\square$ no training \\
\hline 3. How much was the athlete's performance affected by & 4 How severe were the athlete's health problems / \\
injury, illness or other health problems during the past 7 7 & complaints during the past 7 days? \\
days? & $\square$ no symptoms / health problems \\
$\square$ not at all & $\square$ minor \\
$\square$ minor & $\square$ moderate \\
$\square$ moderate & $\square$ severe \\
$\square$ major & $\square$ no training and no competition \\
$\square$ could not participate at all & \\
&
\end{tabular}

Figure 6: Section of team trainers' report that is answered to provide information on the team trainer's perceived severity of the injury or illness.

The team trainer's perceived severity of the injury or illness over the past 7 days was recorded by answering four questions on this reporting form that involved training competition volume changes, the athlete's performance, and the complaints of the athlete (Figures 6 and 7). The team trainer also recorded whether the injury or illness was new, recurrent after full participation, or reported last week (Figure 7).

If the athlete had complaints or any injury, illness or other health problems, please answer the following questions:
\begin{tabular}{|l|l|}
\hline $\begin{array}{l}5 . \text { On how many days did the athlete have complaints } \\
\text { during the past } 7 \text { days? }\end{array}$ & $\begin{array}{l}6 . \text { How many training or competition days did the } \\
\text { athlete miss due to these complaints? }\end{array}$ \\
\hline $\begin{array}{l}\text { 7. Are the complaints new, recurrent or have been } \\
\text { reported last week? }\end{array}$ \\
$\begin{array}{l}\text { new } \\
\text { necurrent after full participation in sport }\end{array}$ \\
\begin{tabular}{l} 
reported last week \\
\hline
\end{tabular}
\end{tabular}

Figure 7: Section of team trainers' report that is completed to provide information on the athlete's complaints and missed training caused by the injury or illness, as well as whether the injury or illness was new, recurrent, or reported in previously. 


\begin{tabular}{|lll|}
\hline$\square$ no treatment & pharmacological \\
$\square$ & rest & $\square$ surgical \\
$\square$ & massage & $\square$ physiotherapy \\
$\square$ & athletic therapy & $\square$ others, specify \\
\hline
\end{tabular}

Figure 8: Section of team trainers' report that is completed to provide information on any treatment the athlete received for the injury or illness.

Lastly, the team trainer reported on whether the athlete received any treatment for the injury or illness in the 7 days following the injury (Figure 8).

\section{Athlete injury and illness reporting form}

The athlete also completed an individual reporting form for each injury or illness that they sustained. This form included information regarding the athlete's training and competition volume changes, their perceived severity, the location of their complaints, the cause or type of their complaints, and any diagnosis of their injury or illness.

Please answer the following questions to provide information on the severity of your injury/illness:

\begin{tabular}{|c|c|}
\hline $\begin{array}{l}\text { 1. Have you had any difficulties in participating in normal } \\
\text { training and competition due to injury, illness or other } \\
\text { health problems during the past } 7 \text { days? } \\
\text { full participation without health problems } \\
\square \text { full participation but with injury / illness } \\
\square \text { reduced participation due to injury / illness } \\
\square \text { could not participate due to injury / illness }\end{array}$ & $\begin{array}{l}\text { 2. To what extent have you modified the volume, content or } \\
\text { intensity of training due to injury, illness or other health } \\
\text { problems during the past } 7 \text { days? } \\
\square \text { no modification of training } \\
\square \text { to a minor extent } \\
\square \text { to a moderate extent } \\
\square \text { to a major extent } \\
\square \text { could not participate at all }\end{array}$ \\
\hline $\begin{array}{l}\text { 3. To what extent have injury, illness or other health } \\
\text { problems affected your performance during the past } 7 \text { days? } \\
\text { no effect } \\
\square \text { to a minor extent } \\
\square \text { to a moderate extent } \\
\square \text { to a major extent } \\
\square \text { could not participate at all }\end{array}$ & $\begin{array}{l}\text { 4. To what extent have you experienced symptoms / health } \\
\text { problems during the past } 7 \text { days? } \\
\square \text { no symptoms / health problems } \\
\square \text { to a minor extent } \\
\square \text { to a moderate extent } \\
\square \text { to a severe extent }\end{array}$ \\
\hline
\end{tabular}

Figure 9: Section of athletes' report that is answered to provide information on the athlete's perceived severity of the injury or illness.

The athlete's perceived severity of the injury or illness over the previous 7 days was recorded by answering four questions on this reporting form that involved training competition volume changes, their performance, and their complaints (Figures 9 and 10). 
The athlete also recorded whether the injury or illness was new, recurrent after full participation, or reported last week (Figure 10).

If you had complaints or any injury, illness or other health problems, please also answer the following questions:
\begin{tabular}{|l|l|}
\hline $\begin{array}{l}\text { 5. On how many days have you had complaints during the } \\
\text { past } 7 \text { days? }\end{array}$ & $\begin{array}{l}\text { 6. How many training or competition days did you miss due } \\
\text { to these complaints? }\end{array}$ \\
\hline $\begin{array}{l}\text { 7. Are the complaints new, recurrent or have been reported } \\
\text { last week? }\end{array}$ \\
$\begin{array}{l}\square \text { new } \\
\text { recurrent after full participation in sport }\end{array}$ \\
\hline$\quad$ reported last week
\end{tabular}

Figure 10: Section of athletes' report that is completed to provide information on their complaints and missed training caused by the injury or illness, as well as whether the injury or illness was new, recurrent, or reported in previously.

\begin{tabular}{|llll|}
\hline 8. Please indicate the location of the complaints referred to above. & \\
\hline$\square$ & head, specify: eye, ear, nose, face, dental & shoulder / clavicle / scapula & $\square$ hip/groin \\
$\square$ neck / cervical spine & $\square$ upper arm & $\square$ thigh \\
$\square$ chest / sternum / rib & $\square$ elbow & $\square$ knee \\
$\square$ thoracic spine / upper back & $\square$ forearm & $\square$ lower leg / Achilles tendon \\
$\square$ lumbar spine / lower back & $\square$ wrist & $\square$ ankle \\
$\square$ abdomen & $\square$ hand foot / toe \\
$\square$ pelvis / sacrum / buttock & $\square$ finger / thumb & $\square$ other, please specify \\
\hline
\end{tabular}

Figure 11: Section of athletes' report that is completed to provide information on the location of the complaints caused by their injury or illness.

The two sections of this reporting form included the athlete's opinion on the bodily location of their complaints, cause of their complaints, type of illness, and any diagnosis of their injury or illness (Figures 11, 12, and 13).

\begin{tabular}{|c|c|c|}
\hline \multirow{2}{*}{$\begin{array}{l}\text { 9. If injury: please indicate the cause of } \\
\text { complaints. }\end{array}$} & \multicolumn{2}{|c|}{ 10. If illness / mental health problem: please indicate the type of illness / complaints. } \\
\hline & 口 asthma & $\square$ headache / migraine \\
\hline$\square$ overuse & diarrheoa, nausea, vomiting & fatigue, lack of energy \\
\hline traumatic non-contact (eg. fall) & otitis (eg. ear infection) & mental health problems \\
\hline traumatic contact with person & allergy (eg. hay fever) & other, please specify: ___ \\
\hline traumatic contact with object/animal & flu, influenza, sinusitis, cold, cough & \\
\hline
\end{tabular}

Figure 12: Section of athletes' report that is completed to provide information on the cause or type of complaints caused by their injury or illness.

11. If a trainer / clinician / healthcare professional made a diagnosis, please report:

Figure 13: Section of athletes' report that is completed to provide information on any diagnosis of their injury or illness. 


\section{Data analysis}

Compliance was calculated as the number of consenting athletes for all teams divided by the total number of athletes on the active team rosters. Similarly, response rate was calculated as the number of forms received from trainers and athletes divided by the number of forms expected for reporting all injuries and illnesses that occurred. Both compliance and response rates were calculated for the individual teams, men and women, and the total cohort.

$\mathrm{AE}$ was calculated in reference to both competition and training, so that injury rates could be presented per $1000 \mathrm{AE}$ exposures (Junge et al 2008). Competition AE for each team was calculated as the maximum number of athletes allowed to compete in the event at one time plus the maximum number of substitutes that can enter a single even multiplied by the number of competitions (or events) that the team participated in during the competition season. Training AE exposure was calculated by multiplying the number of athletes on each team that consented to participate in the study by the number of practices the team had during the competition season. Competitions and training missed due to injury were subtracted from the total AE for each team (Junge et al 2008).

Injury and illness prevalence were calculated for each varsity team and the entire cohort of varsity athletes in the Fall 2016 semester. Prevalence was calculated as the number of athlete injuries or illnesses, divided by the total number of exposed athletes. Descriptive measures such as, weeks injured or ill, location of injury or illness, type of injury or illness, cause of injury or illness, treatment of injury or illness, and training and competition days missed due to injury or illness were all counted and compared, within and between groups. 
Microsoft excel was used to organize and summarize all data. GraphPad Prism was used to complete all 95\% confidence intervals (CI), 1-way Analysis of Variance (ANOVA), and Chi-Squared statistics. Injury and illness prevalence were presented as means with 95\% CI. Injury prevalence was expressed per 100 athletes or per $1000 \mathrm{AE}$, and illness prevalence was expressed per 100 athletes. Average compliance for men and women was compared using a student t-test to examine any differences between averages of men's and women's sports. One-way ANOVA tests were used to compare compliance, rate of response, and injury and illness severity perceived by athletes and trainers between all teams and groups. Chi-Squared tests presented risk ratios (RR) with 95\% CI comparing the mean number of injuries and illnesses between teams and groups of athletes. P values (twotailed) $\leq 0.05$ were considered as significant. 


\section{CHAPTER 4: RESULTS}

Fifteen varsity teams that had their competition season take place in the Fall 2016 semester (September to November) were approached to participate in this research project. Due to less than one third of the consent forms (only 14 of 49 players consented) being returned to the research group from the women's rugby team, these data were only included in the compliance calculation for this study. Of the other 14 varsity teams, only 10 women and 9 men from various team rosters failed to return their consent forms. All other varsity athletes agreed to participate in this research $(86 \%$ of eligible varsity athletes provided consent). Of the 338 participating varsity athletes that were exposed to injury or illness during their competition season, $99(\sim 29 \%)$ were women and $238(\sim 71 \%)$ were men. Competition seasons ranged from six to twelve weeks, with 3110 competition $\mathrm{AE}$ and 12724 training AE (Table 1).

\section{Compliance and rate of response}

The overall compliance from varsity teams was $86 \%$ and ranged from $29 \%$ in women's rugby to $100 \%$ in men's and women's cross country, men's and women's golf, men's football, and men's rowing. The overall compliance was $96 \%$ for men and $72 \%$ for women. When comparing average compliance between men's and women's varsity sports, there was no statistical difference between the two groups $(p=0.252)$ (Figure 14). However, when the compliance of the women's rugby team was left out of the calculation, the compliance for men and women were both above $90 \%$, and the compliance for all athletes was $94 \%$.

All of the team trainers' weekly and individual injury and illness report forms were returned to the researchers for a response rate of $100 \%$ in all varsity sports (Figure 14). 
The athlete rate of response was $100 \%$ for men and $96 \%$ for women (Figure 14). Only two athletes on the women's cross country team failed to return their injury and illness report forms to the researchers. The average of rate of response for all athletes was $98 \%$.

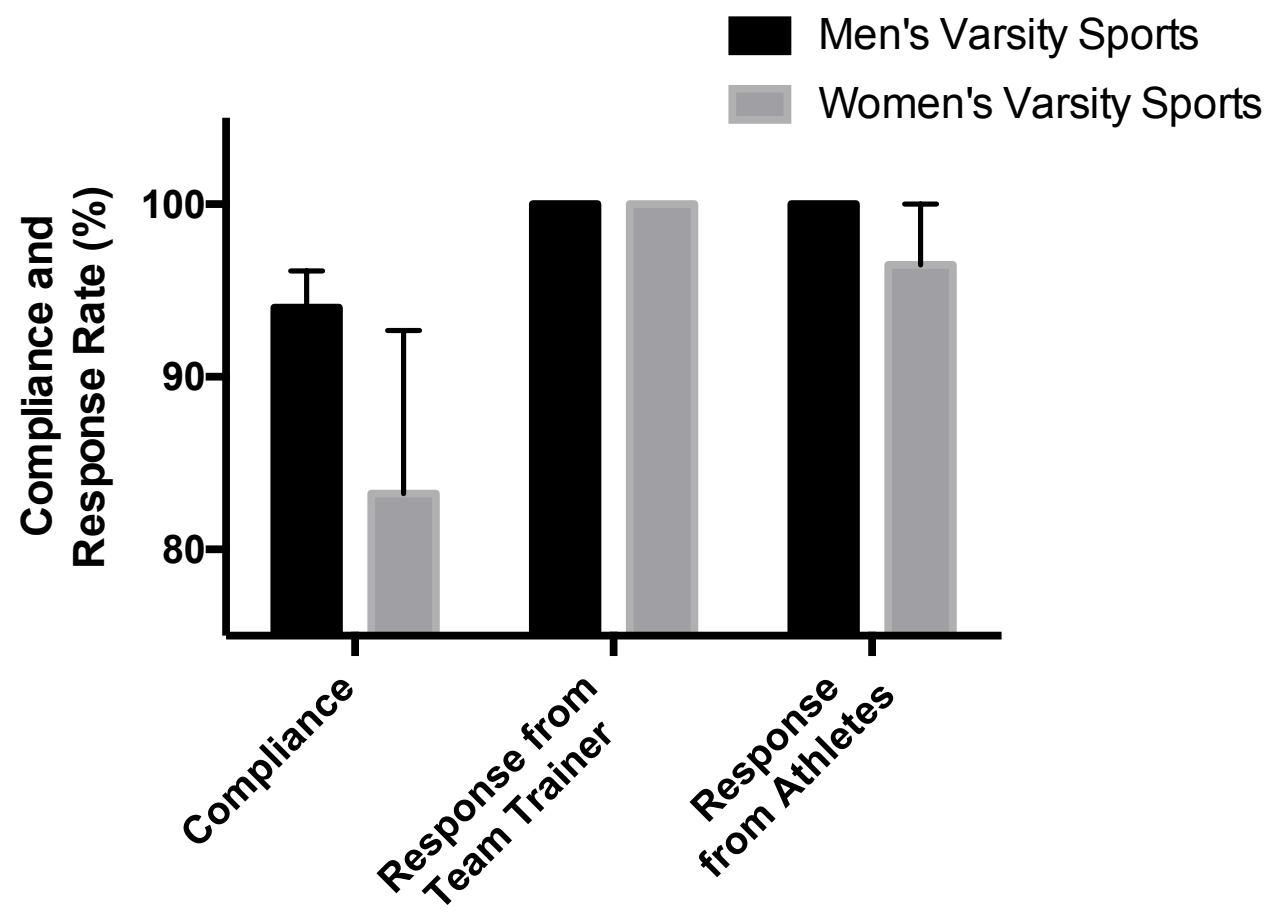

Figure 14: Compliance, and rate of response from team trainers and athletes for implemented varsity athlete injury and illness surveillance protocol for men and women.

\section{Prevalence of injuries}

A total of 142 injuries were recorded from 338 varsity athletes during their competition seasons in the 2016 fall semester, totalling an overall injury rate of 42.0 injuries (95\% CI 34.4 to 49.7 ) per 100 participating athletes. This corresponded to similar injury rates of 9.1 (95\% CI 7.1 to 11.1 ) and 8.7 (95\% CI 5.9 to 11.4) injuries per $1000 \mathrm{AE}$ for men and women, respectively, RR $=1.05$ ( $95 \%$ CI 0.95 to 1.15) (Table 1, Figure 15). In total, 106 athletes were injured with $74 \%(n=78)$ of athletes sustaining one injury, $19 \%(n=20)$ 
of athletes sustaining two injuries, $6 \%(n=6)$ of athletes sustaining three injuries, and $<1 \%$ $(n=1)$ of athletes sustaining four injuries.

When taking exposure into account, an injury rate of 9.0 (95\% CI 7.3 to 10.6) injuries per $1000 \mathrm{AE}$ was found. Sport injury rates ranged from 1.5 (95\% CI -1.6 to 4.6) injuries per 1000 athlete exposures in men's baseball to 30.2 (95\% CI 22.8 to 37.6 ) injuries per 1000 athlete exposures in men's rugby. Men's injury rates were significantly less than women's in cross country ( $R R=0.46(0.41$ to 0.51$))$ and lacrosse $(R R=0.44(0.40$ to 0.49$))$. There was no difference in men's and women's soccer injury rates $(R R=0.95(0.88$ to 1.02)). Men's and women's golf, and men's rowing varsity athletes did not report any injuries (Table 1, Figure 15).

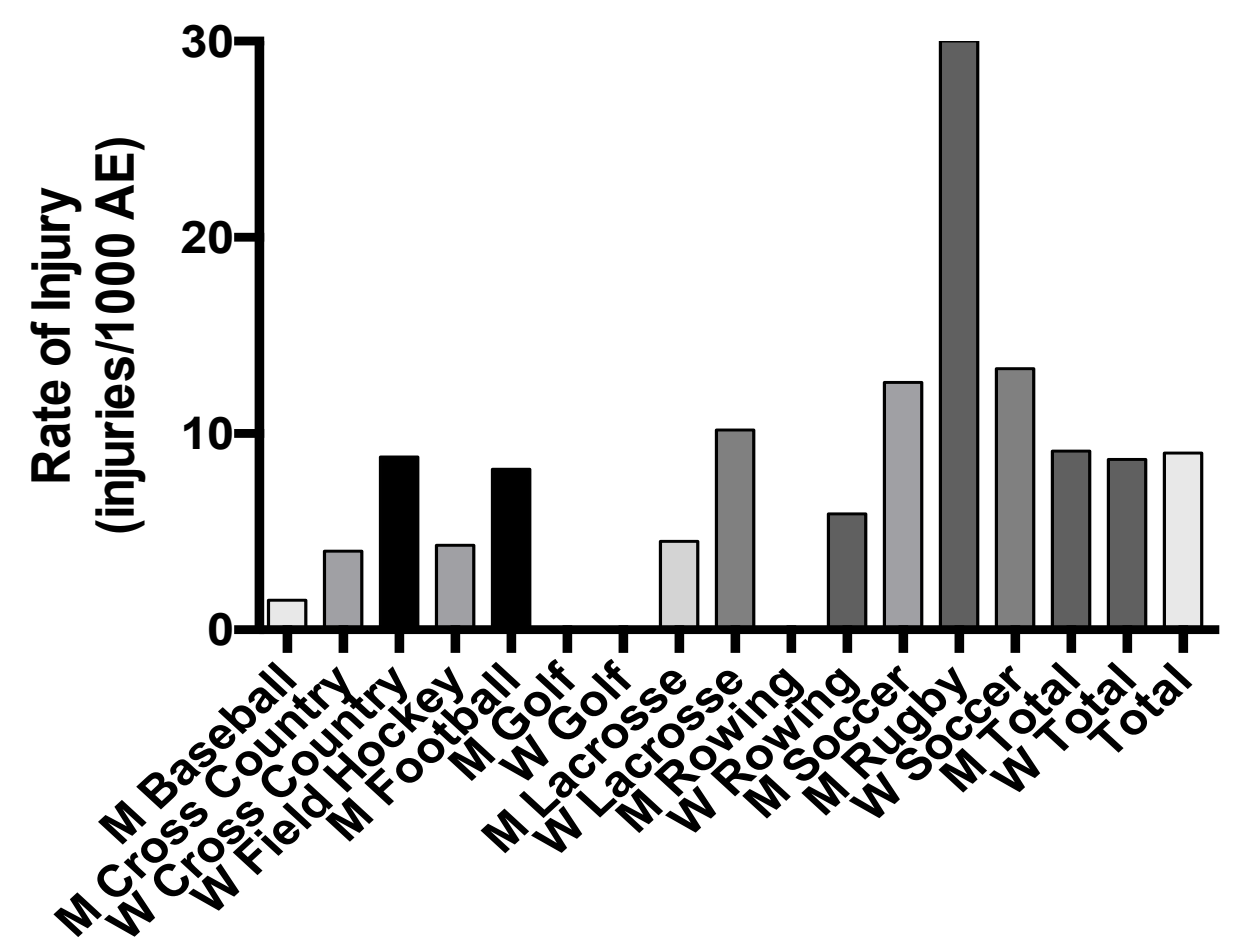

Figure 15: Injury rates of varsity athletes for all teams, men (M), women (W), and athletes. Injury rates in competition (26.4 injuries (95\% CI 20.0 to 32.8$) / 1000 \mathrm{AE}$ ) were higher than in training (4.8 injuries (95\% CI 3.5 to 6.1)/1000 AE), RR = 5.51 (5.00 to 6.07). 
It was also found that injury rates in men's competition (34.9 injuries (95\% CI 25.3 to 44.4)/1000 AE) were higher than in women's competition (12.6 injuries (95\% CI 6.2 to 19.1)/1000 AE), RR = 2.76 (2.59 to 2.95$)$. However, women's injury rate in training (7.2 injuries (95\% CI 4.0 to 10.4$) / 1000 \mathrm{AE}$ ) was greater than the men's injury rate in training (4.0 injuries ( $95 \%$ CI 2.7 to 5.4$) / 1000 \mathrm{AE}$ ), $\mathrm{RR}=0.56$ (0.50 to 0.63 ).

Contact varsity sports (10.6 injuries (95\% CI 8.5 to 12.6$)$ per $1000 \mathrm{AE}$ ) were found to have a higher injury rate compared to non-contact varsity sports (5.5 injuries (95\% CI 3.2 to 7.8 ) per $1000 \mathrm{AE}$ ), $\mathrm{RR}=1.93$ (1.74 to 2.14 ). When looking at gender differences between contact and non-contact sports, men's ( $R R=4.10$ (3.59 to 4.70)) and women's (RR $=1.22$ (1.11 to 1.34$)$ ) injury rates were both greater in contact sports compared to noncontact sports.

Table 1: Number of athletes observed during their competition seasons, and number of injuries, illnesses, competition and training athlete exposures (AE), and lengths of season for each varsity sports team.

\begin{tabular}{lcccccc}
\hline \multicolumn{1}{c}{ Varsity Sport } & $\begin{array}{c}\text { Number } \\
\text { of } \\
\text { Athletes }\end{array}$ & $\begin{array}{c}\text { Number of } \\
\text { Injuries } \\
\text { (injuries/ } \\
\text { 1000 AE) }\end{array}$ & $\begin{array}{c}\text { Number of } \\
\text { Illnesses } \\
\text { (illnesses/ } \\
\text { 100 athletes) }\end{array}$ & $\begin{array}{c}\text { Number of } \\
\text { Athlete } \\
\text { Competition } \\
\text { Exposures } \\
\text { (events) }\end{array}$ & $\begin{array}{c}\text { Number of } \\
\text { Athlete } \\
\text { Training } \\
\text { Exposure } \\
\text { (practices) }\end{array}$ & $\begin{array}{c}\text { Length } \\
\text { of } \\
\text { Season } \\
\text { (weeks) }\end{array}$ \\
\hline $\begin{array}{l}\text { Women's Sports } \\
\text { Cross Country }\end{array}$ & 17 & $8(8.8)$ & $4(23.5)$ & 62 & 843 & 8 \\
Field Hockey & 17 & $3(4.3)$ & $1(5.9)$ & 304 & 389 & 8 \\
Golf & 6 & - & - & 44 & 60 & 6 \\
Lacrosse & 17 & $8(10.2)$ & $4(23.5)$ & 289 & 495 & 7 \\
Rowing & 18 & $5(5.9)$ & $2(11.1)$ & 184 & 661 & 9 \\
Soccer & 24 & $14(13.3)$ & $2(8.3)$ & 306 & 751 & 10 \\
Women's Total & 99 & $38(8.7)$ & $13(13.1)$ & 1189 & 3199 & - \\
Men's Sports & 26 & $1(1.5)$ & - & 360 & 308 & 6 \\
Baseball & 24 & $5(4.0)$ & $6(25.0)$ & 72 & 1167 & 8 \\
Cross Country & 99 & $46(8.2)$ & $8(8.1)$ & 459 & 5186 & 10 \\
Football & 8 & - & $1(12.5)$ & 55 & 79 & 6 \\
Golf & 29 & $5(4.5)$ & $2(6.9)$ & 345 & 766 & 9 \\
Lacrosse & 6 & - & - & 66 & 192 & 7 \\
Rowing & 19 & $29(30.2)$ & $1(5.3)$ & 190 & 770 & 10 \\
Rugby & 28 & $18(12.6)$ & - & 374 & 1057 & 12 \\
Soccer & 239 & $104(9.1)$ & $18(7.5)$ & 1921 & 9525 & - \\
Men's Total & 338 & $142(9.0)$ & $31(9.2)$ & 3110 & 12724 & - \\
Total & & & & & & \\
\hline
\end{tabular}




\section{Location, type, and mechanism of injuries}

Of all injuries, 81 injuries (57\%) occurred during competition and 61 injuries (43\%) occurred during training (Tables 2 - 5). While the majority of athlete's injuries were sustained in the lower extremities (61\%), 30 injuries (21\%) were sustained to the head and trunk, and 25 (18\%) were sustained to the upper extremities. Sprains were the most common type of injury, accounting for $35 \%$ of all injuries. Athletes also sustained 38 (27\%) muscle strains, ruptures, or tears, 13 (9\%) contusions, 13 (9\%) concussions, and 7 (5\%) tendinosis or tendinopathy injuries. More than half (51\%) of all injuries reported were caused by contact with another athlete. Overuse injuries accounted for 35\% of all injuries with 29 injuries (20\%) being classified as gradual overuse injuries and 21 injuries (15\%) being classified as sudden overuse injuries. 
Table 2: Injury location and type, and mechanism of injury during men's varsity competition.

\begin{tabular}{|c|c|c|c|c|c|}
\hline \multirow[b]{2}{*}{ Injury Location } & \multirow[b]{2}{*}{ Type of Injury } & \multicolumn{4}{|c|}{ Mechanism of Injury } \\
\hline & & Overuse & $\begin{array}{c}\text { Non- } \\
\text { Contact } \\
\text { Trauma }\end{array}$ & $\begin{array}{l}\text { Contact } \\
\text { with an } \\
\text { Athlete }\end{array}$ & $\begin{array}{c}\text { Other } \\
\text { Contact }\end{array}$ \\
\hline \multicolumn{6}{|l|}{ Head and Neck } \\
\hline \multirow[t]{3}{*}{ Head } & Concussion & - & - & $7(3.6)$ & - \\
\hline & Contusion/Bruise & - & - & $1(0.5)$ & - \\
\hline & Laceration/Abrasion/ Lesion & - & - & $2(1.0)$ & - \\
\hline \multirow[t]{2}{*}{ Chest/Sternum/Ribs } & Traumatic Fracture & - & - & $1(0.5)$ & - \\
\hline & Dislocation/Subluxation & - & - & $1(0.5)$ & - \\
\hline Upper Back/T-Spine & Strain/Muscle Rupture/Tear & $1(0.5)$ & - & - & - \\
\hline \multicolumn{6}{|l|}{ Upper Extremity } \\
\hline \multirow[t]{2}{*}{ Shoulder } & Dislocation/Subluxation & - & - & $2(1.04)$ & - \\
\hline & Sprain & - & - & $14(7.3)$ & $1(0.5)$ \\
\hline Elbow & Sprain & - & - & $2(1.0)$ & - \\
\hline \multirow{2}{*}{\multicolumn{6}{|c|}{ Lower Extremity }} \\
\hline & & & & & \\
\hline Hip/Groin & Strain/Muscle Rupture/Tear & $1(0.5)$ & - & - & - \\
\hline \multirow[t]{2}{*}{ Thigh } & Contusion/Bruise & - & - & $2(1.0)$ & - \\
\hline & Strain/Muscle Rupture/Tear & $3(1.6)$ & - & $1(0.5)$ & - \\
\hline \multirow[t]{2}{*}{ Knee } & Lesion of Meniscus/Cartilage & - & - & $1(0.5)$ & - \\
\hline & Sprain & $1(0.5)$ & - & $5(2.6)$ & $1(0.5)$ \\
\hline \multirow[t]{2}{*}{ Lower Leg/Achilles } & Contusion/Bruise & - & - & $1(0.5)$ & - \\
\hline & Strain/Muscle Rupture/Tear & - & - & $1(0.5)$ & - \\
\hline \multirow[t]{2}{*}{ Ankle } & Sprain & $1(0.5)$ & - & $9(4.7)$ & $1(0.5)$ \\
\hline & Tendon Rupture & - & $1(0.5)$ & - & - \\
\hline \multirow[t]{3}{*}{ Foot/Toes } & Laceration/Abrasion/Lesion & $1(0.5)$ & - & - & - \\
\hline & Ligamentous & - & - & $1(0.5)$ & - \\
\hline & Strain/Muscle Rupture/Tear & $1(0.5)$ & - & - & - \\
\hline Total & & $9(4.7)$ & $1(0.5)$ & $53(27.6)$ & $3(1.6)$ \\
\hline
\end{tabular}

Values presented are numbers (and prevalence rates per 1000 athlete exposures (AE) in brackets) of injured athletes. 
Table 3: Injury location and type, and mechanism of injury during men's varsity training.

\begin{tabular}{|c|c|c|c|c|c|}
\hline \multirow[b]{2}{*}{ Injury Location } & \multirow[b]{2}{*}{ Type of Injury } & \multicolumn{4}{|c|}{ Mechanism of Injury } \\
\hline & & Overuse & $\begin{array}{c}\text { Non- } \\
\text { Contact } \\
\text { Trauma }\end{array}$ & $\begin{array}{l}\text { Contact } \\
\text { with an } \\
\text { Athlete }\end{array}$ & $\begin{array}{c}\text { Other } \\
\text { Contact }\end{array}$ \\
\hline \multicolumn{6}{|l|}{ Head and Neck } \\
\hline Head & Concussion & - & - & $3(0.3)$ & - \\
\hline Lower Back/L-Spine & Strain/Muscle Rupture/Tear & $1(0.1)$ & - & - & - \\
\hline Abdomen & Strain/Muscle Rupture/Tear & - & - & $1(0.1)$ & - \\
\hline Pelvis/Sacrum/Buttock & Ligamentous & $1(0.1)$ & - & - & - \\
\hline \multicolumn{6}{|l|}{ Upper Extremity } \\
\hline \multirow{2}{*}{ Shoulder } & Sprain & - & - & $1(0.1)$ & - \\
\hline & Strain/Muscle Rupture/Tear & - & - & $1(0.1)$ & - \\
\hline Finger/Thumb & Sprain & - & - & - & $1(0.1)$ \\
\hline \multicolumn{6}{|l|}{ Lower Extremity } \\
\hline \multirow[t]{2}{*}{ Hip/Groin } & Contusion/Bruise & - & - & $1(0.1)$ & - \\
\hline & Strain/Muscle Rupture/Tear & $6(0.6)$ & - & - & - \\
\hline Thigh & Strain/Muscle Rupture/Tear & $5(0.5)$ & - & - & - \\
\hline \multirow[t]{3}{*}{ Knee } & Dislocation/Subluxation & - & - & $1(0.1)$ & - \\
\hline & Contusion/Bruise & - & - & $2(0.2)$ & - \\
\hline & Arthritis/Synovitis/Bursitis & - & - & - & $1(0.1)$ \\
\hline \multirow[t]{3}{*}{ Lower Leg/Achilles } & Ligamentous & - & - & $1(0.1)$ & - \\
\hline & Strain/Muscle Rupture/Tear & $2(0.2)$ & - & - & - \\
\hline & Tendinosis/Tendinopathy & $1(0.1)$ & - & - & - \\
\hline Ankle & Sprain & - & $1(0.1)$ & $2(0.2)$ & $1(0.1)$ \\
\hline \multirow[t]{5}{*}{ Foot/Toes } & Contusion/Bruise & - & - & $1(0.1)$ & - \\
\hline & Fasciitis/Aponeurosis Injury & - & $1(0.1)$ & - & - \\
\hline & Laceration/Abrasion/Lesion & $1(0.1)$ & - & - & - \\
\hline & Sprain & $1(0.1)$ & - & - & - \\
\hline & Stress Fracture & $1(0.1)$ & - & - & - \\
\hline Total & & $19(2.0)$ & $2(0.2)$ & $14(1.5)$ & $3(0.3)$ \\
\hline
\end{tabular}

\footnotetext{
Values presented are numbers (and prevalence rates per 1000 athlete exposures (AE) in brackets) of injured athletes.
}

Table 4: Injury location and type, and mechanism of injury during women's varsity competition.

\begin{tabular}{|c|c|c|c|c|c|}
\hline \multirow[b]{2}{*}{ Injury Location } & \multirow[b]{2}{*}{ Type of Injury } & \multicolumn{4}{|c|}{ Mechanism of Injury } \\
\hline & & Overuse & $\begin{array}{c}\text { Non- } \\
\text { Contact } \\
\text { Trauma }\end{array}$ & $\begin{array}{l}\text { Contact } \\
\text { with an } \\
\text { Athlete }\end{array}$ & $\begin{array}{l}\text { Other } \\
\text { Contact }\end{array}$ \\
\hline \multicolumn{6}{|l|}{ Head and Neck } \\
\hline Head & Concussion & - & - & - & $2(1.7)$ \\
\hline $\begin{array}{l}\text { Lower Back/L-Spine } \\
\text { Upper Extremity }\end{array}$ & Strain/Muscle Rupture/Tear & $1(0.8)$ & - & - & - \\
\hline Elbow & Contusion/Bruise & - & - & $1(0.8)$ & - \\
\hline Hip/Groin & Strain/Muscle Rupture/Tear & $1(0.8)$ & - & - & - \\
\hline \multirow[t]{2}{*}{ Thigh } & Contusion/Bruise & - & - & $1(0.8)$ & - \\
\hline & Strain/Muscle Rupture/Tear & $2(1.7)$ & - & - & - \\
\hline Knee & Sprain & - & - & - & $1(0.8)$ \\
\hline Lower Leg/Achilles & Contusion/Bruise & - & - & $1(0.8)$ & - \\
\hline \multirow[t]{3}{*}{ Ankle } & Contusion/Bruise & $1(0.8)$ & - & - & - \\
\hline & Dislocation/Subluxation & - & - & $1(0.8)$ & - \\
\hline & Sprain & - & $3(2.5)$ & - & - \\
\hline Total & & $5(4.2)$ & $3(2.5)$ & $4(3.4)$ & $3(2.5)$ \\
\hline
\end{tabular}

Values presented are numbers (and prevalence rates per 1000 athlete exposures (AE) in brackets) of injured athletes. 
Table 5: Injury location and type, and mechanism of injury during women's varsity training.

\begin{tabular}{|c|c|c|c|c|c|}
\hline \multirow[b]{2}{*}{ Injury Location } & \multirow[b]{2}{*}{ Type of Injury } & \multicolumn{4}{|c|}{ Mechanism of Injury } \\
\hline & & Overuse & $\begin{array}{c}\text { Non- } \\
\text { Contact } \\
\text { Trauma }\end{array}$ & $\begin{array}{l}\text { Contact } \\
\text { with an } \\
\text { Athlete }\end{array}$ & $\begin{array}{l}\text { Other } \\
\text { Contact }\end{array}$ \\
\hline \multicolumn{6}{|l|}{ Head and Trunk } \\
\hline \multirow[t]{2}{*}{ Head } & Concussion & - & - & - & $1(0.3)$ \\
\hline & Contusion/Bruise & - & - & - & $1(0.3)$ \\
\hline Neck/C-Spine & Strain & $1(0.3)$ & - & - & - \\
\hline Upper Back/T-Spine & Strain/Muscle Rupture/Tear & $1(0.3)$ & - & - & - \\
\hline Lower Back/L-Spine & Strain/Muscle Rupture/Tear & $2(0.6)$ & - & - & - \\
\hline Pelvis/Sacrum/Buttock & Strain/Muscle Rupture/Tear & $1(0.3)$ & - & - & - \\
\hline \multicolumn{6}{|l|}{ Upper Extremity } \\
\hline Wrist & Strain/Muscle Rupture/Tear & $1(0.3)$ & - & - & - \\
\hline Finger/Thumb & Sprain & - & - & - & $1(0.3)$ \\
\hline \multicolumn{6}{|l|}{ Lower Extremity } \\
\hline Hip/Groin & Strain/Muscle Rupture/Tear & $1(0.3)$ & - & - & - \\
\hline Thigh & Strain/Muscle Rupture/Tear & $1(0.3)$ & - & - & - \\
\hline Knee & Tendinosis/Tendinopathy & $3(0.9)$ & - & - & - \\
\hline Lower Leg/Achilles & Strain/Muscle Rupture/Tear & $3(0.9)$ & - & - & - \\
\hline Ankle & Sprain & - & $2(0.6)$ & - & - \\
\hline \multirow{2}{*}{ Foot/Toes } & Fasciitis/Aponeurosis Injury & $1(0.3)$ & - & - & - \\
\hline & Tendinosis/Tendinopathy & $3(0.9)$ & - & - & - \\
\hline Total & & $18(5.6)$ & $2(0.6)$ & $0(0.0)$ & $3(0.9)$ \\
\hline
\end{tabular}

The only injury reported for men's baseball was a shoulder dislocation. The injury rates for men's and women's cross country were 4.0 injuries (95\% CI -0.1 to 8.2 ) and 8.8 injuries (95\% CI 3.9 to 13.8 ) per 1000 AE. All 12 of the injuries in men's and women's cross country athletes were classified as lower extremity overuse injuries that occurred in training. Men's cross country athletes sustained four strains and one tendinosis injury, while women's cross country athletes sustained four tendinosis injuries and three muscle strain injuries. Women's field hockey athletes reported two injuries (one thigh bruise and one ankle sprain) that occurred in competition and one injury (head contusion) that occurred in training.

The injury rate for men's football was 8.2 injuries (95\% CI 5.4 to 10.9) per $1000 \mathrm{AE}$, respectively. Of the 46 injuries (32\% of all injuries) reported by men's football athletes, 28 
(61\%) injuries occurred in competition and 18 (39\%) injuries occurred in training. Competition injuries consisted of ten ankle sprains, five sprains in the knee, four concussions, four sprains in the shoulder region, two thigh muscle strains, one hand fracture, one rib fracture, and one Achilles tendon rupture. The mechanism of $93 \%$ of these injuries was contact with another athlete. Training injuries consisted of three concussions, three ankle sprains, three hamstring strains, three muscle strains in the hip region, one sprain in the shoulder region, one shoulder muscle strain, one abdomen muscle strain, one contusion in the hip region, one knee contusion, and one patellar dislocation. Eleven (61\%) of these injuries were the result of contact with another athlete and six (33\%) of these injuries were considered overuse injuries.

The injury rates for men's and women's lacrosse were 4.5 injuries (95\% CI 0.7 to 8.3) and 10.2 injuries (95\% CI 0.5 to 20.0) per $1000 \mathrm{AE}$, respectively. Men's lacrosse athletes sustained $80 \%$ of their reported injuries in competition. Three of these injuries were the result of contact with another athlete and the other two injuries were considered overuse injuries. These injuries were comprised of one concussion, one sprain in the shoulder region, one knee sprain, and one lower leg muscle rupture. The reported training injury was a groin strain. Women's lacrosse athletes sustained five injuries in competition and three injuries in training. Less than $20 \%$ of these injuries were the result of contact with another athlete. Competition injuries consisted of two ankle sprains, one bruised ankle, one bruised elbow, and one lower back strain. Training injuries consisted of two knee tendinosis injuries and one sprained ankle. All five injuries that occurred in women's rowing were classified as overuse injuries that occurred in training. These injuries 
consisted of two lower back strains, one upper back strain, one wrist strain, and one bruised foot.

Men's rugby athletes had the highest injury rate (30.2 injuries (95\% CI 22.8 to 37.6 ) per $1000 \mathrm{AE}$ ) of all sports. Competition injuries accounted for $90 \%$ of the injuries reported for this sport, which consisted of nine sprains in the shoulder region, three ankle sprains, two concussions, two elbow sprains, two head lacerations, two hamstring strains, and one muscle strain in the foot, shoulder dislocation, rib subluxation, knee sprain, head contusion, and thigh contusion. Contact with an athlete was the mechanism of injury for $77 \%$ of these injuries.

The injury rate for men's soccer athletes was 12.6 injuries (95\% CI 6.7 to 18.5$)$ per 1000 AE. Thirty-nine percent of their injuries were sustained in competition and $61 \%$ of their injuries in training. Competition injuries consisted of two back muscle strains, a hip muscle strain, a thigh contusion, a knee sprain, a toe skin lesion, and a toe ligamentous injury. Fifty-seven percent of these injuries resulted from contact with an athlete or the ball, while the rest were considered overuse injuries. Training injuries consisted of a lower back muscle strain, a hip ligamentous injury, a hip muscle strain, a thigh muscle strain, a lower leg ligamentous injury, an ankle sprain, a bruised foot, a foot skin lesion, a stress fracture in the foot, a plantar fasciitis injury, and a toe sprain. The majority (64\%) of these injuries were considered overuse injuries.

The injury rate for women's soccer athletes was 13.3 injuries (95\% CI 7.0 to 19.5 ) per 1000 AE. These athletes sustained $57 \%$ of their injuries during competition and $43 \%$ of their injuries during training. Competition injuries consisted of two thigh muscle strains, two concussions, one hip flexor strain, one knee sprain, one bruised lower leg, and one 
ankle dislocation. Sixty-three percent of these injuries were a result of contact with an athlete or the ground while the rest were considered overuse injuries. Training injuries consisted of one concussion, one neck muscle strain, one thumb sprain, one hip muscle strain, one lower leg muscle strain, and one ankle sprain. Four of these injuries were considered overuse injuries and two of these injuries were the result of contact with the ball.

\section{Severity of injuries}

Of all 142 injuries, $96(68 \%)$ injuries caused the athlete to miss at least one training day, and $18(13 \%)$ of those injuries caused the athlete to miss more than seven days of training (Table 6). Fifty-eight of these injuries caused the athlete to miss at least one competition, and only one of these injuries caused the athlete to miss more than seven competitions. The average time missed due to injury for all varsity athletes was 3.1 (95\% CI 2.4 to 2.9 ) full training days and 0.8 (95\% CI 0.6 to 1.0$)$ competitions. It was found that men (6.6 injuries (95\% CI 5.3 to 7.8 ) per $1000 \mathrm{AE}$ ) had a higher risk of sustaining an injury that forced them to miss at least one training day compared to women (4.8 injuries (95\% CI 2.9 to 6.6) per $1000 \mathrm{AE}$ ), $\mathrm{RR}=1.37$ (1.22 to 1.54 ). Injuries that resulted in at least one day of training loss in male athletes ranged from 1.5 injuries (95\% CI -1.6 to 4.6) per $1000 \mathrm{AE}$ in baseball to 10.5 injuries (95\% CI 6.6 to 14.3 ) per $1000 \mathrm{AE}$ in soccer. Injuries that resulted in at least one day of training loss for female athletes ranged from 1.2 injuries (95\% CI -1.3 to 3.7) per $1000 \mathrm{AE}$ in rowing to 8.5 injuries (95\% CI 3.8 to 13.3 ) per $1000 \mathrm{AE}$ in soccer.

When comparing rates of injury severity by competition-time lost, it was found that men's injuries (3.9 injuries ( $95 \%$ CI 2.9 to 5.0 ) per $1000 \mathrm{AE}$ ) were more likely to force the athlete to miss at least one competition (event) compared to women's injuries (3.0 injuries 
( $95 \% \mathrm{CI} 1.4$ to 4.5 ) per $1000 \mathrm{AE}$ ), RR = 1.32 (1.14 to 1.54 ). Injuries that required men to miss at least one competition ranged from 1.5 injuries (95\% CI -1.6 to 4.6) per $1000 \mathrm{AE}$ in baseball to 9.4 injuries (95\% CI 4.5 to 14.3 ) per $1000 \mathrm{AE}$ in rugby. Injuries that required women to miss at least one competition ranged from 1.2 injuries (95\% CI -1.3 to 3.7$)$ per $1000 \mathrm{AE}$ in rowing to 7.6 injuries (95\% $\mathrm{CI} 3.0$ to 12.2$)$ per $1000 \mathrm{AE}$ in soccer.

Numerous statistical differences were found when comparing injury severity questionnaires that were completed by team trainers and athletes. When assessing complaints to team trainers by athletes, there were significantly more days of complaints in men's football versus men's soccer ( $p=0.03$ ), men's football versus women's field hockey $(\mathrm{p}<0.04)$, men's lacrosse versus women's field hockey $(\mathrm{p}=0.04)$, women's lacrosse versus women's field hockey ( $p=0.02)$, women's rowing versus women's field hockey $(p=0.01)$, and women's soccer versus women's field hockey $(\mathrm{p}=0.01)$. The athletes claimed that they had a more difficulty participating in training and competition in men's football, men's lacrosse, men's rugby, men's soccer, women's cross country, and women's soccer compared to women's field hockey ( $\mathrm{p}$ values $<0.04$ ). Athletes in men's football and men's lacrosse also had significantly higher scores than women's field hockey when expressing their extent of injury symptom severity and their days of complaints while injured ( $p$ values $<0.05$ ). However there were no significant differences in perceived injury severity scores when comparing responses of athletes and team trainers (Figure 16). 


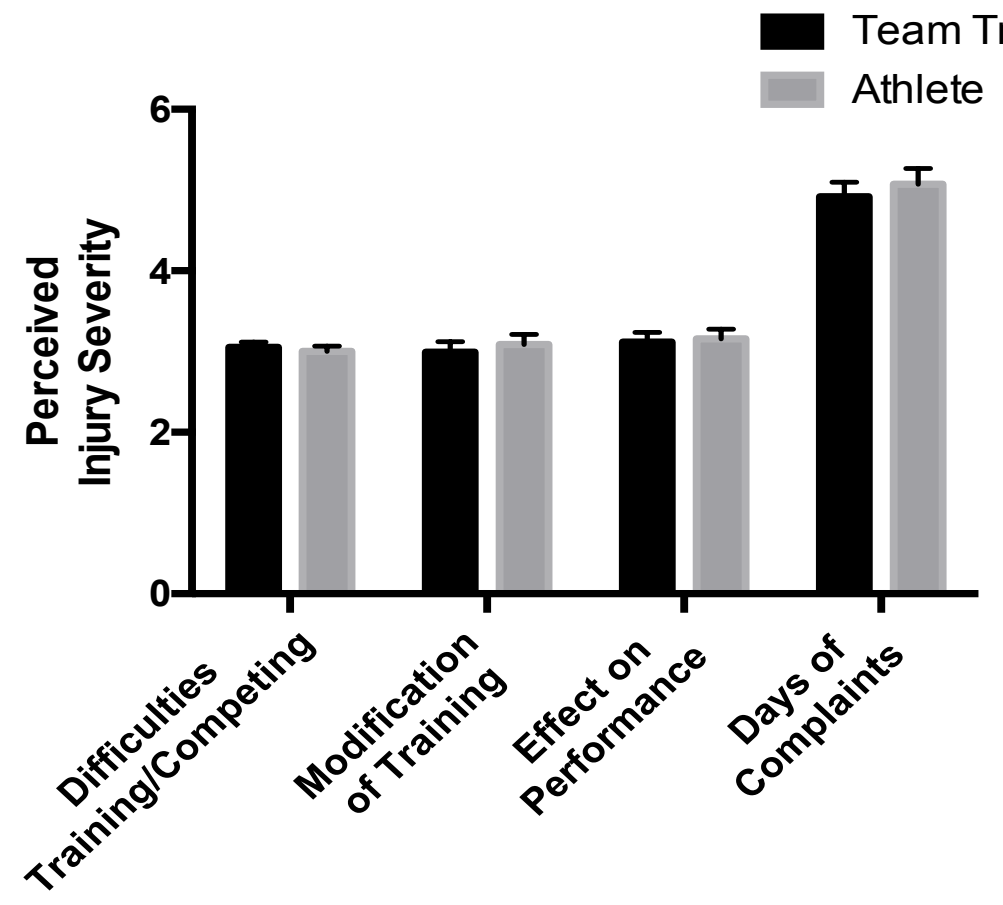

Figure 16: Average injury severity questions completed by athletes and team trainers.

Table 6: Men's and women's injury severity represented by training and competition days missed.

\begin{tabular}{lcccccc}
\hline \multicolumn{1}{c}{ Sport } & \multicolumn{3}{c}{ Full Training Missed } & \multicolumn{3}{c}{ Competition Missed } \\
\cline { 2 - 7 } & $\begin{array}{c}0 \\
\text { days }\end{array}$ & $\begin{array}{c}1-7 \\
\text { days }\end{array}$ & $8-28$ days & $\begin{array}{c}0 \\
\text { event }\end{array}$ & $\begin{array}{c}1-7 \\
\text { events }\end{array}$ & $\begin{array}{c}8-28 \\
\text { events }\end{array}$ \\
\hline WOMEN & & & & & & \\
Cross Country & $2(2.2)$ & $5(5.5)$ & $1(1.1)$ & $5(5.5)$ & $3(3.3)$ & - \\
Field Hockey & $2(2.9)$ & $1(1.4)$ & - & $3(4.3)$ & - & - \\
Golf & - & - & - & - & - & - \\
Lacrosse & $4(5.1)$ & $4(5.1)$ & - & $7(8.9)$ & $1(1.3)$ & - \\
Rowing & $4(4.7)$ & $1(1.2)$ & - & $4(4.7)$ & $1(1.2)$ & - \\
Soccer & $5(4.7)$ & $7(6.6)$ & $2(1.9)$ & $6(5.7)$ & $8(7.6)$ & - \\
Women's Total & $17(3.9)$ & $18(4.1)$ & $3(0.7)$ & $25(5.7)$ & $13(3.0)$ & - \\
MEN & & & & & & \\
Baseball & - & $1(1.5)$ & - & - & $1(1.5)$ & - \\
Cross Country & $1(0.8)$ & $3(2.4)$ & $1(0.8)$ & $4(3.2)$ & $1(0.8)$ & - \\
Football & $6(1.1)$ & $31(5.5)$ & $9(1.6)$ & $24(4.3)$ & $22(3.9)$ & - \\
Golf & - & - & - & - & - & - \\
Lacrosse & - & $5(4.5)$ & - & $1(0.9)$ & $4(3.6)$ & - \\
Rowing & - & - & - & - & - & - \\
Rugby & $19(19.8)$ & $9(9.4)$ & $1(1.0)$ & $20(20.8)$ & $9(9.4)$ & - \\
Soccer & $3(2.1)$ & $11(7.7)$ & $4(2.8)$ & $10(7.0)$ & $7(4.9)$ & $1(0.7)$ \\
Men's Total & $29(2.5)$ & $60(5.2)$ & $15(1.3)$ & $59(5.2)$ & $44(3.8)$ & $1(0.1)$ \\
ToTALS & $46(2.9)$ & $78(4.9)$ & $18(1.1)$ & $84(5.3)$ & $57(3.6)$ & $1(0.1)$ \\
\hline
\end{tabular}

Values presented are numbers (and prevalence rates per 1000 athlete exposures (AE) in brackets) of injured athletes. 
Men had a significantly greater score when trainers rated their modification of training volume $(\mathrm{p}=0.03)$ and their performance decrements $(\mathrm{p}=0.03)$ as a result of their injuries compared to women. When comparing contact and non-contact sports, team trainers reported injured athletes in contact sports to have a greater difficulty participating in normal training and competition $(\mathrm{p}=0.03)$, greater modifications of their training volume $(\mathrm{p}<0.01)$, greater performance decrements $(\mathrm{p}<0.01)$, and more severe complaints in relation to their injury $(\mathrm{p}<0.01)$ compared to athletes in non-contact sports. These differences were also found when comparing contact and non-contact sports within men, however not within women. The athlete's perception of difficulty participating in training and competition $(\mathrm{p}<0.01)$, and modification of their training volume $(\mathrm{p}<0.01)$ was also significantly worse in contact sports compared to non-contact sports.

\section{Treatment of injuries}

Varsity athletes reported a total of 245 injury treatments during their competition season. Only three injuries received no treatment. The most common type of treatment for all athletes was athletic therapy (41\%), followed by rest $(23 \%)$ and treatment from a sports medicine doctor (10\%) (Table 7). When separating men's and women's injury treatments, AT was still the most common treatment for both groups (men: 39\%, women: 48\%). The lone injured men's baseball athlete received treatment of rest, AT, and physiotherapy. The most common treatment for men's cross country athletes was massage therapy (43\%), followed by physiotherapy (29\%) and AT (14\%). However, women's cross country athletes most common treatments for injuries were physiotherapy and rest (31\%). Rest was the most common treatment for injuries in men's soccer (30\%) and women's field hockey (60\%), while AT was the most common treatment for injuries in men's football 
(47\%), men's (44\%) and women's (75\%) lacrosse, men's rugby (42\%), women's rowing (56\%), and women's soccer (62\%).

Table 7: Treatment of injuries for men and women varsity athletes reported by team trainers.

\begin{tabular}{lccc}
\hline \multicolumn{1}{c}{ Treatment } & Men & Women & Total \\
\hline No Treatment & - & 3 & 3 \\
Rest & 49 & 8 & 57 \\
Massage Therapy & 9 & 3 & 12 \\
Athletic Therapy & 74 & 27 & 101 \\
Physiotherapy & 12 & 8 & 20 \\
Chiropractic & - & 1 & 1 \\
Sports Medicine & 20 & 5 & 25 \\
Imaging & 2 & 1 & 3 \\
Surgical & 1 & - & 1 \\
Pharmacological & 5 & 1 & 6 \\
Acupuncture & 0 & 2 & 2 \\
Bracing/Taping & 16 & - & 16 \\
Sutures/Staples & 1 & - & 1 \\
\hline
\end{tabular}

\section{Prevalence of illnesses}

A total of 31 illnesses were recorded from 338 varsity athletes during their competition seasons in the 2016 fall semester, totalling an overall illness rate of 9.2 illnesses (95\% CI 6.1 to 12.3 ) per 100 participating athletes. This corresponded to an illness rate of $7.5(95 \%$ CI 4.2 to 10.90$)$ per 100 athletes for men that was lower than the women's illness rate of 13.1 (95\% CI 6.4 to 19.9$)$ illnesses per 100 athletes, $\mathrm{RR}=0.57(0.53$ to 0.62 ) (Table 1, Table 8, Figure 17). In total, 29 athletes reported an illness with 28 of these athletes acquiring one illness and one of these athletes acquiring three illnesses. 


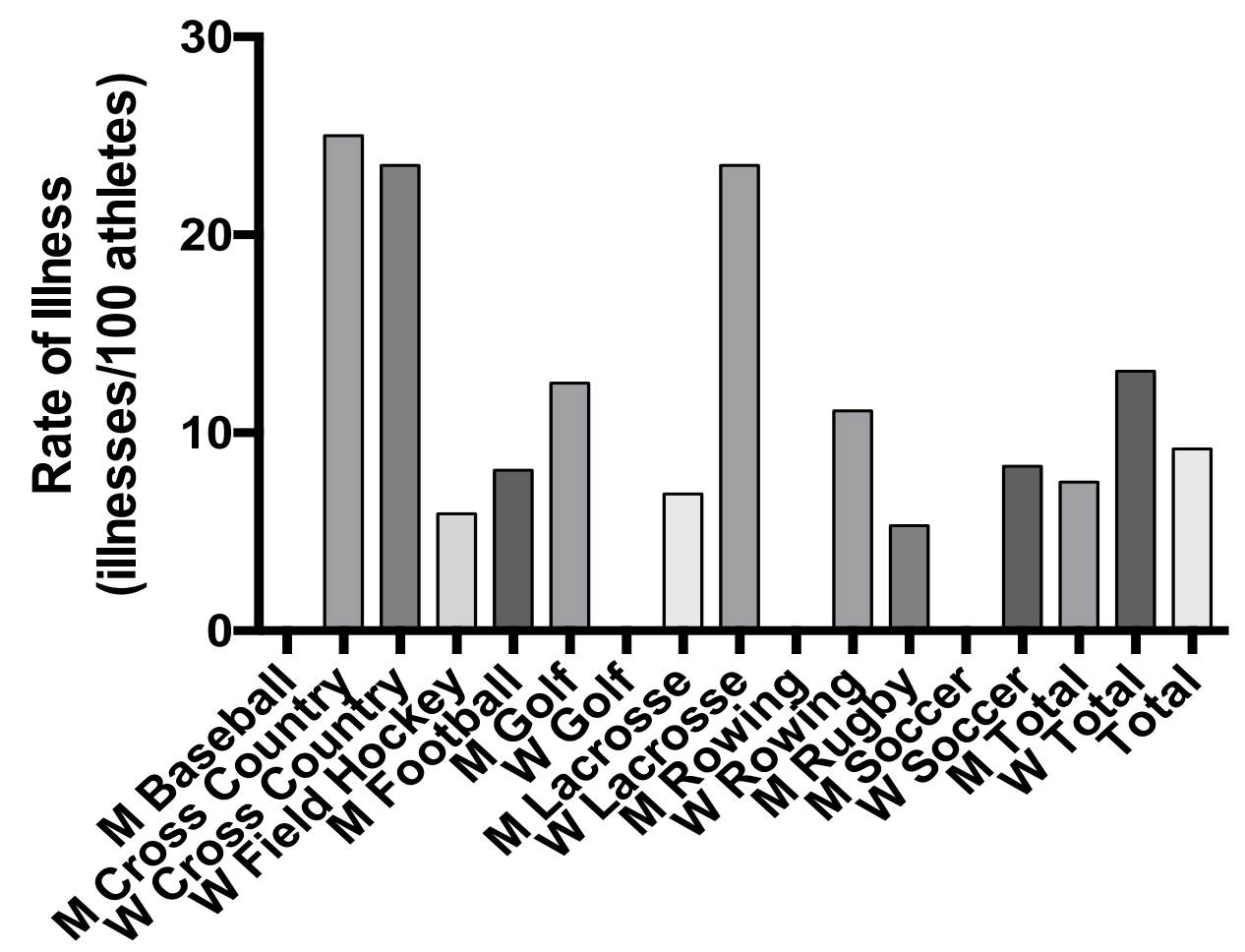

Figure 17: Illness rates of varsity athletes for all teams, men (M), women (W), and athletes. Illness rates for men's individual teams were 25.0 illnesses (95\% CI -3.5 to 53.5) per 100 athletes for cross country, 8.1 illnesses (95\% CI 2.6 to 13.5) per 100 athletes for football, 12.5 illnesses (95\% CI -17.1 to 42.1) per 100 athletes for golf, 6.9 illnesses (95\% CI -2.9 to 16.7) per 100 athletes for lacrosse, and 5.3 illnesses (95\% CI -5.8 to 16.3) per 100 athletes for rugby. Illness rates for women's individual teams were 23.5 illnesses (95\% CI 1.1 to 46.0 ) per 100 athletes for cross country, 5.9 illnesses (95\% CI -6.6 to 18.4) per 100 athletes for field hockey, 23.5 illnesses (95\% CI 1.1 to 46.0) per 100 athletes for lacrosse, 11.1 illnesses (95\% CI -5.0 to 27.2) per 100 athletes for rowing, 8.3 illnesses (95\% CI -3.4 to 20.3) per 100 athletes for soccer. Men's baseball, men's rowing, men's soccer, and women's golf athletes had no reported illnesses. 


\section{Affected body system and diagnosis of illnesses}

The most common body system affected by illness was found to be the upper respiratory tract system (65\% of illnesses), followed by the gastrointestinal system (GI) (19\%), the neurological or central nervous system (10\%), and the lower respiratory system (6\%) (Table 8). Men's and women's cross country accounted for almost one third (32\%) of the total illnesses recorded. Of the ten illnesses recorded from these two sports, seven were considered Rhinopharyngitis, one was the flu, one was GI distress, and one with bronchitis. The Rhinopharyngitis and bronchitis illnesses were caused by infection while the GI distress was exercise induced, and a virus resulted in the flu for one athlete.

Illnesses from the men's football athletes accounted for $26 \%$ of all illnesses. Five of these eight illnesses were recorded as streptococcus pharyngitis caused by an infection. An infection was also the cause of a bronchitis illness suffered by one athlete. Another athlete experienced the flu virus and one athlete suffered from vasovagal presyncope, from an unknown cause.

Women's field hockey, men's golf, and men's rugby athletes only reported one illness each that consisted of a fever, an upper respiratory tract infection, and a streptococcus pharyngitis, respectively. All three of these illnesses were caused by an infection. Men's and women's lacrosse athletes accounted for 19\% of all illnesses, with three athletes experiencing the flu virus, two upper respiratory tract infections, and one athlete suffering from Bell's Palsy. Women's rowing and women's soccer athletes each only suffered two infections causing three upper and one lower respiratory tract infections between the two groups. 
Table 8: Men's and women's affected body system and diagnosis of illnesses during their competition season.

\begin{tabular}{lccc}
\hline \multicolumn{1}{c}{ Affected System/Illness Diagnosis } & Men & Women & Total \\
\hline Upper Respiratory Tract System & $6(2.5)$ & $8(8.1)$ & $14(4.1)$ \\
$\begin{array}{l}\text { Rhinopharyngitis } \\
\text { Streptococcus Pharyngitis (strep } \\
\text { throat) }\end{array}$ & $6(2.5)$ & - & $6(1.8)$ \\
$\begin{array}{l}\text { Lower Respiratory Tract System } \\
\text { Bronchitis }\end{array}$ & $1(0.4)$ & $1(1.0)$ & $2(0.6)$ \\
Gastrointestinal System & & & \\
Flu & $2(0.8)$ & $3(3.0)$ & $5(1.5)$ \\
GI Distress & $1(0.4)$ & - & $1(0.3)$ \\
Neurological/Central Nervous System & & & $1(0.3)$ \\
Bell's Palsy & $1(0.4)$ & - & $1(0.3)$ \\
Fever & - & $1(1.0)$ & $1(0.3)$ \\
Vassovagal Presyncope & $1(0.4)$ & - & $31(9.2)$ \\
Total & $18(7.5)$ & $13(13.1)$ & \\
\hline Values presented are numbers (and prevar & & & \\
\hline
\end{tabular}

Values presented are numbers (and prevalence rates per 100 athletes in brackets) of ill athletes.

\section{Severity of illnesses}

The prevalence rates of acquiring an illness that resulted in an athlete time loss of at least one training day or competition were 6.8 illnesses (95\% CI 4.1 to 9.5) per 100 athletes and 3.0 illnesses (95\% CI 1.1 to 4.8) per 100 athletes, respectively. Prevalence rates for training days missed were greater in men (5.4 illnesses (95\% CI 2.5 to 8.3 ) per 100 athletes) than women (4.2 illnesses (95\% CI -4.5 to 12.8 ) per 100 athletes), RR $=1.30(1.15$ to 1.48). Similarly, prevalence rates for competitions missed were significantly different between men (3.8 illnesses (95\% CI 1.3 to 6.2) per 100 athletes) and women (1.0 illnesses (95\% CI -1.0 to 3.0) per 100 athletes), $\mathrm{RR}=3.73$ (3.00 to 4.64). Of all 38 illnesses, 23 illnesses caused the athlete to miss at least one training day and only one illness required 
the athlete to miss more than seven days of training. Only ten illnesses caused athletes to miss competition and no athlete missed more than three competition days due to illness. When comparing illness severity questions that were completed by team trainers and athletes, there was no difference in responses between teams, team trainers and athletes, or men and women.

\section{Treatment of illnesses}

Of the 31 recorded illnesses, athletes received 37 illness treatments. Only four illnesses received no treatment. Rest was found to be the most common treatment (38\%) for athletes that were ill, followed by intervention from a sports medicine doctor (30\%), pharmacological treatment (30\%), and athletic therapy (2\%) (Table 9).

Table 9: Treatment of illnesses for men and women varsity athletes reported by team trainers.

\begin{tabular}{lccc}
\hline \multicolumn{1}{c}{ Treatment } & Men & Women & Total \\
\hline No Treatment & - & 4 & 4 \\
Rest & 10 & 8 & 14 \\
Athletic Therapy & 1 & - & 1 \\
Sports Medicine & 8 & 3 & 11 \\
Pharmacological & 7 & 4 & 11 \\
\hline
\end{tabular}




\section{CHAPTER 5: DISCUSSION}

\section{Overview}

The main purpose of this research was to implement an injury and illness surveillance protocol in varsity athletes at a Canadian university in Guelph, ON during their competition season. The second purpose of this research was to identify injury and illness characteristics in various sports with the goal that the information learned would inform future injury prevention measures with optimal athlete health in mind. The main findings of this research were that of 338 athletes (239 men and 99 women), 31\% suffered an injury (42.0 injuries per 100 participating athletes, or 9.0 injuries per $1000 \mathrm{AE}$ ) and $8 \%$ of athletes reported an illness (9.2 illnesses per 100 participating athletes) during their competition season. There were many significant differences between sports regarding injury and illness characteristics.

Of the total 142 injuries reported, 104 (73\%) injuries occurred in men and 38 (27\%) injuries occurred in women. Although there were more injuries in men than women, injury rates (9.1 and 8.7 injuries per $1000 \mathrm{AE}$ for men and women, respectively) were found to be statistically similar when athlete exposure was taken into account, which differed from the hypothesis. Conversely, illness rates were found to be significantly lower in men (7.5 per 100 athletes) than women (13.1 illnesses per 100 athletes).

Men's football athletes suffered the most injuries of any team (32\% of all injuries), followed by men's rugby (20\%), men's soccer (13\%), women's soccer $(10 \%)$, women's cross country (6\%), women's lacrosse (6\%), men's cross country (3\%), men's lacrosse (3\%), women's rowing (3\%), women's field hockey $(2 \%)$, and men's baseball $(<1 \%)$. Similarly, men's football reported the most illnesses during their competition season $(26 \%$ 
of all 31 illnesses reported), followed by men's cross country (19\%), women's cross country $(13 \%)$, women's lacrosse $(13 \%)$, women's rowing $(6 \%)$, women's soccer $(6 \%)$, men's lacrosse (6\%), women's field hockey (3\%) men's golf (3\%), and men's rugby (3\%).

Another important finding is that a prospective injury and illness surveillance protocol, like the one used in this study, was feasible to implement and was widely accepted by athletes and team trainers in a Canadian university, given the $96 \%$ and $75 \%$ compliance rates for men and women, respectively. Although, the women's rate fell below our hypothesis of reaching $80 \%$ compliance, the compliance rate of $86 \%$ for all participants reached the expected level. Only the women's varsity rugby team, and nine men and ten women from other varsity teams chose not to participate in the research. The rates of response from team trainers and athletes were also above $96 \%$.

\section{Injury rates in collegiate and university sport}

To our knowledge, this is the first study to report on implementation of prospective injury and illness surveillance in all varsity athletes at a Canadian university. For this reason, it is not feasible to compare overall injury and illness rates with other findings in other Canadian university sports. However, there is one 5-year epidemiological study that examined injury surveillance of CIS football players in Western Canadian Universities (Meeuwisse et al. 2000). There are also several other studies at the NCAA and collegiate levels that at least allow for comparison of injury rates within specific sports (Agel et al. 2007, Dick et al. 2007b,c,d,e,f, Kerr et al. 2008, Kerr et al. 2016). It is important to note that sport rules, athlete skill, and injury surveillance protocols are not all the same between studies, which likely has some effect on differences within sports. 
Only one baseball injury was recorded in this study and it occurred during competition. This competition injury rate (2.8 injuries per $1000 \mathrm{AE}$ ) was lower than the competition injury rate (5.7 injuries per 1000 AE) reported in the 2003-2004 NCAA baseball season (Dick et al. 2007f). In cross country, men's injury rates (4.0 injuries per $1000 \mathrm{AE}$ ) were significantly lower than and women's (8.8 injuries per $1000 \mathrm{AE}$ ), which followed a similar trend to those reported in the NCAA (4.7 injuries per $1000 \mathrm{AE}$ for men 5.9 injuries per $1000 \mathrm{AE}$ for women) (Kerr et al. 2016). As you can see, men's injury rates in this study were similar to our findings but the women's injury rates found in our study were higher than what was found in the NCAA.

Women's field hockey injury rates in the present study were 6.6 injuries per 1000 $\mathrm{AE}$ in competition and 2.6 injuries per $1000 \mathrm{AE}$ in training. When comparing these injury rates to those of the 2002-2003 NCAA season (6.6 per $1000 \mathrm{AE}$ in competition and 3.6 per $1000 \mathrm{AE}$ in training), the competition injury rates were found to be very similar but the training injury rate in the NCAA was higher (Dick et al. 2007b). Meeuwisse and colleagues (2000), performed one of the only studies that reported on CIS sport to date, in football players of western Canadian universities from 1993-1997. This retrospective study reported that in 1997, the injury rate of these football players was 20.1 per $1000 \mathrm{AE}$. This injury rate is double the injury rate (8.2 injuries per $1000 \mathrm{AE})$ that the present results show (Meeuwisse et al. 2000).

Men's lacrosse injury rates were 11.6 per $1000 \mathrm{AE}$ in competition and 1.3 injuries per $1000 \mathrm{AE}$ in training. The competition injury rate was very similar to the competition injury rate from the 2003-2004 NCAA men's lacrosse season (12.0 injuries per $1000 \mathrm{AE}$ ) (Dick et al. 2007e). However, the training injury rate in the NCAA that year (3.7 injuries per 
$1000 \mathrm{AE}$ ) was greater than what the present results showed. Women's lacrosse injuries rates followed the same trend and were 17.3 injuries per $1000 \mathrm{AE}$ for competition and 6.1 injuries per $1000 \mathrm{AE}$ for training. Our findings were much higher than injury rates reported in the 2003-2004 NCAA women's lacrosse team in both competition (6.2 injuries per 1000 AE) and training (2.9 injuries per $1000 \mathrm{AE}$ ) (Dick et al. 2007c). Differences in injury surveillance protocols, between Canadian and USA lacrosse rules, and the development of the sport are all possible reasons for discrepancies between data.

There are no injury surveillance studies specific to rowing athletes at the collegiate level, but several studies have reported a low injury rate for the sport of rowing at other levels (Engebretsen et al. 2013, Smoljanovic et al. 2011, Yang et al. 2012). One study that looked at acute and overuse injuries in NCAA athletes found that out of 16 sports, women's rowing had the most overuse injuries (Yang et al. 2012). The present results follow the same trend with all five of the injuries sustained by women's rowing athletes occurring during training and being classified as overuse injuries. Together these data outline the importance of future work focusing on training programs and other interventions to reduce overuse injuries.

Men's rugby had the highest injury rate during the competition season. However, it is important to mention that only the "first-string" players were monitored in this research, which could inflate injury rates. The competition injury rate was 136.8 injuries per 1000 $\mathrm{AE}$ and the training injury rate was 3.9 injuries per $1000 \mathrm{AE}$. The competition injury rate was substantially higher than in a 2005-2006 collegiate rugby union study in the USA (22.5 injuries per $1000 \mathrm{AE}$ ) (Kerr et al. 2008). However, the training injury rate for this collegiate study (5.5 injuries per $1000 \mathrm{AE}$ ) was only slightly higher than the current work. 
Men's soccer injury rates were 21.4 injuries per $1000 \mathrm{AE}$ for competition and 10.4 injuries per $1000 \mathrm{AE}$ for training and were greater than competition (18.4 injuries per $1000 \mathrm{AE}$ ) and training (4.5 injuries per $1000 \mathrm{AE}$ ) injury rates found in the 2002-2003 NCAA men's soccer season (Agel et al. 2007). However, both results followed the same trend of their being more injuries in competition than training. Women soccer injury rates followed the same trend, while competition and training injury rates were 26.1 and 8.0 injuries per $1000 \mathrm{AE}$, respectively. Other studies have reported lower injury rates of women's soccer players ranging from 16.4 to 19.0 injuries per $1000 \mathrm{AE}$ in competition and 4.8 to 5.2 injuries per $1000 \mathrm{AE}$ in training (Dick et al. 2007d; Yang et al. 2012).

Although some injury rates from our study are very similar to those reported in the NCAA, there are many differences. In addition to the fact that differences likely occurred due to different leagues and different rules, there are a few other reasons that should be considered to explain these differences. The studies used for comparison were chosen because of the limited data in injury surveillance in collegiate and university sport. However, many of these studies presented data from more than a decade ago, which could account for many possible differences given changes of rules, equipment, and education to athletes and coaches (Agel et al. 2007, Dick et al. 2007b,c,d,e,f, Engebretsen et al. 2013, Kerr et al. 2008). Many of these studies also had a much larger sample size as they recorded information from dozens of schools across three divisions of the NCAA. Lastly, one of the most significant differences to consider is that the majority of the NCAA studies used the NCAA ISS to record injury information, which is a different method of collection than what was used in this research (Agel et al. 2007, Dick et al. 2007b,c,d,e,f, Kerr et al. 2008, Kerr et al. 2016). The biggest difference between protocols is that the current study used 
prospective analysis with team trainer and athlete reporting rather than a standard injury database reporting mechanism. Comparison between injury rates will likely provide some insight on the matter but without using the same protocol for injury surveillance, there will likely always be some differences in results.

\section{Location, type, and mechanism of injuries}

It was hypothesized that contact sports would have greater injury rates than noncontact sports. As expected, the injury rate of 10.6 injuries per $1000 \mathrm{AE}$ for contact sports was greater than the injury rate of 5.5 per $1000 \mathrm{AE}$ for non-contact sports. This is not surprising, given that $51 \%$ of all injuries occurred from contact with another athlete. Similarly, the fact that fifty-seven percent of all injuries occurred in competition agreed with our hypothesis that more injuries would occur in competition than in training. Only men's and women's cross country, and women's rowing had more injuries that occurred in training compared to competition, which was also supported by previous retrospective literature (Engebretsen et al. 2013, Kerr et al. 2016, Smoljanovic et al. 2011, Yang et al. 2012). Given the fact that these sports have a very high AE to training compared to competition, these results are not surprising. Interventions specific to overuse injuries should be introduced as prevention measures moving forward.

Many of the studies that reported on injuries in the sports considered in this research, found that lower extremity injuries were most common in athletes (Agel et al. 2007, Dick et al. 2007b,c,d,e, Kerr et al. 2016). Our results also followed this trend, with $61 \%$ of all injuries occurring in the lower extremities of athletes. Men's football and rugby athletes had previously reported more head injuries than any other location, however our results showed that the lower extremities were the most common location of football 
injuries and the upper extremities were the most common location of men's rugby injuries (Kerr et al. 2008, Meeuwisse et al. 2000). Women's rowing injuries had been reported to occur mostly in the lower back and upper extremities, which were consistent with the results found in our research (Engebretsen et al. 2013, Smoljanovic et al. 2011, Yang et al. 2012).

The most common types of injuries were ligament sprains (35\% of all injuries), followed by muscle (strains, ruptures, or tears) contusions, and concussions. Sprains of the ankle, knee, and shoulder were the most common injuries that occurred in football, which was different than the most common injury of concussions that was previously reported (Meeuwisse et al. 2000). Men's rugby also followed this trend (45\% of all men's rugby injuries), with athletes sustaining nine sprains in the shoulder, three ankle sprains, and one sprain in the knee.

Contact with another athlete was the most common mechanism of injury for men's football competition and training, men's lacrosse, rugby and soccer competitions, and women's soccer competition. These findings are consistent with previous injury information within these sports (Agel et al. 2007, Dick et al. 2007c,d, Kerr et al. 2008, Meeuwisse et al. 2000). Overuse injuries were most common in men's and women's cross country training, men's and women's lacrosse training, women's rowing training, and men's and women's soccer training. The sports that were most affected by overuse injuries were men's and women's cross country, and women's rowing, as all of their injuries occurred in training and were considered to be caused by overuse. 


\section{Severity of injuries}

There are many ways to rate severity of injuries but the most common rating mechanism is by recording time loss from training and competition. This method of assessing severity is logical, as the majority of elite athletes have a high drive to play at all costs, and injuries are one of the only things that can keep them from training, competing, and performing at their best. Thirty-two percent and 59\% of injuries did not require athletes to miss any training and/or competition, respectively. However, 55\% of injuries required athletes to miss between one and seven days of training and $13 \%$ of injuries required athletes to miss between 8 and 28 days of training. In addition to this, $40 \%$ of injuries required the athlete to miss between one and seven competitions and only one $(<1 \%)$ injury required an athlete to miss more than seven competitions.

Although concussions were not the most prevalent injury, given the cognitive effects of this injury in a university population, these injuries are of particular interest and importance in varsity athletes' health throughout the competition season. Of the twelve concussions reported, eight occurred in competition and four occurred during training. Men's football athletes sustained seven concussions, men's rugby athletes sustained two concussions, women's soccer athletes sustained two concussions, and one concussion was sustained in men's lacrosse. Concussions caused athletes to miss 70 days of training and 23 competitions in just twelve different cases. Four of these injuries caused men's football athletes to miss more than 15 days of training. Previous research recording concussion injuries in high school and collegiate athletes supported our findings (Gessel et al. 2007). Results showed that the majority in concussions occurred from participating in football compared to other sports such as baseball, basketball, soccer, and volleyball. 
The average for days of training missed was 3.1 days and the average for competitions missed was 0.8 competitions. When comparing teams, football had the highest average for training days missed (5.0 days) and baseball had the highest average for competitions missed (3.0 competitions) for men. Cross country had the highest average for training days missed (2.9 days) and soccer had the highest average for competitions missed (1.2 competitions) for women. One very interesting thing to note here is that although men's rugby had the highest injury rate, it had one of the lowest averages for days of training (1.5 days) and competitions (0.4 competitions) missed. Given the prevalence and types of injuries sustained by these athletes, this finding illustrates that these athletes train and compete even while injured. This phenomenon is supported in the literature and demonstrates the limitation of using time loss from sport as an indicator of athlete health (Mountjoy 2015a).

Another interesting way that the severity of injuries was assessed with this protocol was through the perception of both the team trainer and the athlete by asking them to answer questions based on training and competition volume, performance, and complaints. Complaints were found to be more severe from men's football team trainers and athletes compared to several other sports, which is supported by the time-loss findings. Women's field hockey team trainers and athletes had less severe responses than most sports, however this team only had three injuries in total. Men's severity scores were found to be greater than women's when injured, and athletes in contact sports reported greater severity scores than those in non-contact sports. Assessing severity in more than one way allows for greater comparison once prevention measures are tested. 


\section{Illness risk in Canadian university sport}

There is no research presenting illness surveillance in varsity athletes from Canadian Universities, nor in collegiate sports in the NCAA. The overall illness rate for varsity athletes was 9.17 illnesses per 100 athletes. Although there were more illnesses in men than women in absolute terms, illness rates per 100 athletes were higher in women than men. These trends of illness prevalence have been supported in World Championships and the Olympic Games (Alonso et al. 2010, Mountjoy et al. 2010, Soligard et al. 2014).

Severity of illnesses was measured by time-loss from training and competition. Only one illness (bronchitis in men's football) impacted the athletes training and competition in a substantial manner, resulting in 17 days away from training and from three competitions. Although illnesses were asked to be reported regardless of severity or time loss, these results demonstrate that tracking illnesses in athletes may be more variable than tracking injuries, likely due to individual variability of symptoms and athlete perception. Only ten of the fourteen varsity teams reported at least one illness, but it is unclear whether this could be due to underreporting. Through further contact directly with athletes about complaints rather than diagnosis, it is possible that underreporting could be minimized (Clarsen et al. 2013).

\section{Methodological considerations and practical implications}

In studies measuring sports injury epidemiology, it is most common and effective to express injury rates in reference to exposures because it allows for comparison between teams with different volumes of training and competition (Bahr and Holme 2003, Derman et al. 2013, Fuller et al. 2006, Schwellnus et al. 2013, Willick et al. 2013). Although we were able to approach teams after the season to at least estimate the frequencies of the training 
and competition exposure for each sport so that injuries could be expressed in injuries per $1000 \mathrm{AE}$, capturing exposure prospectively would likely improve accuracy and reduce recall bias. Without modification to the protocol, training and competition length were not able to be collected to express injuries per AE hours. Conversely, due to the fact illnesses were measured regardless of whether or not they were obtained because of training or competition, we felt the best representation of comparison between teams was by presenting them as illnesses per 100 athletes.

Using consistent definitions for injuries and illnesses, we were able to optimize a standard for data collection. However, despite pre-season training and regular periodic support from the research team, it is important to note that there is variability between team trainers and athletes in regards to reporting injuries and illnesses. Although $32 \%$ and $59 \%$ of injuries required no training or competition loss, it is possible that less severe injuries went undetected if they do not require medical attention (Finch 1997, Inklaar 1994). The only way to correct for this would involve daily or weekly reporting directly from athletes about any complaints, even if they do not consider themselves to be injured (Clarsen et al. 2013). One challenge for athlete injury and illness diagnosis and management at this Canadian University is that not all varsity teams were assigned a team trainer for the year. The men's and women's cross country team and the men's and women's golf team were able to find their own team trainers, while women's lacrosse and men's and women's rowing did not have a team trainer. For the purpose of this study, our research team was able to collect data for these teams by interacting with athletes before and after training sessions. Although these athletes were able to visit the Health and 
Performance Centre on campus whenever they needed treatment, it is still concerning that these athletes do not have their own team trainer to provide athlete health support.

With very high compliance and rates of response from team trainers and athletes, this injury and illness surveillance protocol was able to create a baseline database of injuries and illnesses for varsity athletes in various sports. Now that this baseline data has been recorded, it is recommended that sport- and injury-specific prevention measures be implemented to athletes before executing the same protocol to test for differences. Participants from all teams, except the men's and women's cross country team, filled out reports in paper form. Given the amount of work that this injury and illness protocol requires from our research team, the team trainers, and the athletes, it is recommended that the protocol should be able to be completed electronically. It would also allow for the checking of duplication of data in case team trainers and other health care professionals at the campus Health and Performance Centre want to enter data into the same database. This would help minimize the possibility of injuries and illnesses going unreported.

\section{Limitations}

As with any human research, there were limitations that should be taken into account when considering the results of this study. First, injuries and illnesses were only recorded during the competition season, which neglected athletes that were injured during the offseason and training camp. These pre-season injuries could potentially render athletes more susceptible to injuries during the season or time-lost at the start of the season before they are fully recovered. After discussion with team coaches, trainers, and athletes, it was clear that the training and competition volume is much higher in training camp, which is a risk factor for more injuries. Although the implementation of athlete 
surveillance during training camps is challenging due to the changing of rosters while teams are being finalized, the capture of this data would likely have provided valuable information on injury prevalence and risk.

Secondly, exposure was estimated following the study by looking at weekly schedules, league rules, and contacting team trainers or coaches. Although our calculations followed previous research for estimation of exposure, it would still be more accurate if exposures were recorded daily for athletes (Junge et al. 2008). This would eliminate recall bias and training or competition exposures that athletes missed for reasons other than injuries or illnesses. This is more important for larger teams that might have numerous extra players that do not play in every competition.

Lastly, the majority of the implementation of the athlete surveillance protocol was completed in paper form. In the future, implementing a similar protocol electronically would allow for easier collection of data, less data input for researchers, more consistent reporting of information, and data input from multiple sources if athletes report injuries and illnesses to the Health and Performance Centre on campus rather than to their team trainers. This method has been used at World Championships and the Olympic Games where athlete injury and illness collection is also obtained form the local organizing committee health services to minimize underreporting (Engebretsen et al. 2010, Engebretsen et al. 2013, Soligard et al. 2015, Prien et al. 2016). Although our research team consulted with the Health and Performance Centre on campus if there were any discrepancies in data, there is still a chance that some athletes reported their injuries and illnesses to other health care professionals other than their team trainers. The protocol was successful in collecting relevant information in regards to athlete injuries and illnesses, 
however having a more standardized, concise, and electronic way of implementing the protocol would also provide quicker feedback to team medical staff so that they can act in response in real-time during the competition season.

\section{Conclusion}

In conclusion, implementing this injury and illness surveillance protocol was considered a success, as indicated by compliance and rate of responses from team trainers and athletes of at least $86 \%$. Thirty-one percent of athletes suffered an injury during their competition season, resulting an injury rate of 42.0 injuries per 100 participating athletes or 9.0 injuries per $1000 \mathrm{AE}$. Although men sustained more injuries than women, their injury rates were not significantly different, while injury rates were found to be significantly greater in contact sports compared to non-contact sports. The illness rate for varsity athletes was 9.2 illnesses per 100 participating athletes with $8 \%$ of athletes reporting an illness. This is the first research that had implemented prospective injury and illness surveillance in varsity athletes at a Canadian university and more research is needed to confirm our findings. However, this study has now set a baseline at the University of Guelph for implementing sport-specific prevention measures and pre-season screening for varsity athletes and their medical staff. 


\section{REFERENCES}

Agel, J., Evans, T.A., Dick, R., Putukian, M., and Marshall, S.W. 2007. Descriptive epidemiology of collegiate men's soccer injuries: National Collegiate Athletic Association Injury Surveillance System, 1988-1989 Through 2002-2003. J. Athl. Train. 42(2):270-277.

Alonso, J. M., Edouard, P., Fischetto, G., Adams, B., Depiesse, F., \& Mountjoy, M. 2012. Determination of future prevention strategies in elite track and field: analysis of Daegu 2011 IAAF Championships injuries and illnesses surveillance. Br J Sports Med. 46:505-514.

Alonso, J. M., Tscholl, P. M., Engebretsen, L., Mountjoy, M., Dvorak, J., and Junge, A. 2010. Occurrences of injuries and illnesses at the 2009 IAAF Championships injuries. $\mathrm{Br} \mathrm{J}$ Sports Med. 44:1100-5.

Bahr R, Holme I. 2003. Risk factors for sports injuries-a methodological approach. $\mathrm{Br} \mathrm{J}$ Sports Med. 37:384-92.

Bahr, R., and Krosshaug, T. 2005. Understanding injury mechanisms: a key component of preventing injuries in sport. Br. J. Sports Med. 39(6):324-9.

Batt, M.E. 1992. A survey of golf injuries in amature golfers. Br. J. Sports Med. 26(1):63-65.

Bere T, Alonso JM, Wangensteen A, et al. 2015a. Injury and illness surveillance during the 24th Men's Handball World Championship 2015 in Qatar. Br J Sports Med. 49(17):1151-6.

Bere T, Kruczynski J, Veintimilla N, et al. 2015b. Injury risk is low among world-class volleyball players: 4-year data from the FIVB Injury Surveillance System. Br J Sports Med. 49(17):1132-7.

Borowski, L. a, Yard, E.E., Fields, S.K., and Comstock, R.D. 2008. The epidemiology of US high school basketball injuries, 2005-2007. Am. J. Sports Med. 36(12):2328-35.

Brooks, J.H.M., and Fuller, C.W. 2006. The Influence of Methodological Issues on the Results and Conclusions from Epidemiological Studies of Sports Injuries. Sport. Med. 36(6): 459-472.

Chambers, C.C., Lynch, T.S., Gibbs, D.B., Ghodasra, J.H., Sahota, S., Franke, K., Mack, C.D., and Nuber, G.W. 2016. Superior Labrum Anterior-Posterior Tears in the National Football League. Am. J. Sports Med. 45(1):167-172.

Clarsen, B., Grethe, M., and Bahr, R. 2013. Development and validation of a new method for the registration of overuse injuries in sports injury epidemiology: the Oslo Sports Trauma Research Centre (OSTRC) Overuse Injury Questionnaire. Br J Sports Med. 495502. 
Collins, C.C.L., and Comstock, R.D. 2008. Epidemiological features of high school baseball injuries in the United States, 2005-2007. Pediatrics. 121(6):1181-1187.

Comstock, D.R., Knox, C., Yard, E., and Gilchrist, J. 2006. Sports-related injuries amoung high school athletes - United States, 2005-06 school year. JAMA J. Am. Med. Assoc. 296(22):3-4.

Derman, W., Schwellnus, M., Jordaan, E., Blauwet, C. A., Emery, C., Pit-Grosheide, P., Marques, N.-A.P., Martinez-Ferrer, O., Stomphorst, J., Van de Vliet, P., Webborn, N., and Willick, S.E. 2013. Illness and injury in athletes during the competition period at the London 2012 Paralympic Games: development and implementation of a web-based surveillance system (WEB-IISS) for team medical staff. Br. J. Sports Med. 47:420-5.

Derman W, Schwellnus M, Jordaan E, et al. 2013. Illness and injury in athletes during the 47 competition period at the London 2012 Paralympic Games: development and implementation of a web-based surveillance system (WEB-IISS) for team medical staff. Br J Sports Med. 47:420-5.

Dick, R., Agel, J., and Marshall, S.W. 2007a. NCAA Injury Surveillance System Commentaries : Introduction and Methods. J. Athl. Train. 42(2):173-182.

Dick, R., Hootman, J.M., Agel, J., Vela, L., Marshall, S.W., and Messina, R. 2007b. Descriptive Epidemiology of Collegiate Women's Field Hockey Injuries: National Collegiate Athletic Association Injury Surveillance System, 1988-1989 Through 2002-2003. J. Athl. Train. 42(2):211-220.

Dick, R., Lincoln, A., Agel, J., Carter, E.A., Marshall, S.W., and Hinton, R.Y. 2007c. Descriptive Epidemiology of Collegiate Women's Lacrosse Injuries: National Collegiate Athletic Association Injury Surveillance System, 1988-1989 Through 2003-2004. J. Athl. Train. 42(2):262-269.

Dick, R., Putikian, M., Agel, J., Evans, T.A., and Marshall, S.W. 2007d. Descriptive Epidemiology of Collegiate Women's Soccer Injuries: National Collegiate Athletic Association Injury Surveillance System, 1988-1989 Through 2002-2003. J. Athl. Train. 42(2):278-285.

Dick, R., Romani, W.A., Agel, J., Case, J.G., and Marshall, S.W. 2007e. Descriptive Epidemiology of Collegiate Men's Lacrosse Injuries: National Collegiate Athletic Association Injury Surveillance System, 1988-1989 Through 2003-2004. J. Athl. Train. 42(2):255-261.

Dick, R., Sauers, E.L., Agel, J., Keuter, G., Marshall, S.W., McCarty, K., and McFarland, E. $2007 f$. Descriptive Epidemiology of Collegiate Men's Baseball Injuries : National Collegiate Athletic Association Injury Surveillance System , 1988 - 1989 Through 2003 - 2004. J. Athl. Train. 42(2):183-193. 
Dompier, T.P., Marshall, S.W., Kerr, Z.Y., and Hayden, R. 2015. The National Athletic Treatment, Injury and Outcomes Network (NATION): Methods of the surveillance program, 2011-2012 through 2013-2014. J. Athl. Train. 50(8): 862-869.

Elliott, M., Zarins, B., Powell, J.W., and Kenyon, C.D. 2011. Hamstring Muscle Strains in Professional Football Players A 10-Year Review. Am. J. Sports Med. 39(4): 843-850.

Emery, C. a, Meeuwisse, W.H., and Hartmann, S.E. 2005. Evaluation of risk factors for injury in adolescent soccer: implementation and validation of an injury surveillance system. Am. J. Sports Med. 33(12):1882-1891.

Emery, C., and Meeuwisse, W.H. 2006. Injury Rates, Risk Factors, and Mechanisms of Injury in Minor Hockey. Am. J. Sports Med. 34(12):1960-1969.

Engebretsen, L., Steffen, K., Alonso, J.M., Aubry, M., Dvorak, J., Junge, A., Meeuwisse, W., Mountjoy, M., Renström, P., and Wilkinson, M. 2010. Sports injuries and illnesses during the Winter Olympic Games 2010. Br. J. Sports Med. 44(11): 772-780.

Engebretsen, L., Steffen, K., Alonso, J.M., Aubry, M., Dvorak, J., Junge, A., Meeuwisse, W., Mountjoy, M., Renström, P., and Wilkinson, M. 2013. Sports injuries and illnesses during the London Summer Olympic Games 2012. Br. J. Sports Med. 44(11): 772-780.

Feddermann-Demont N, Junge A, Edouard P, et al. 2014. Injuries in 13 international Athletics championships between 2007-2012. Br J Sports Med. 48:513-22.

Finch, C.F. 1997. An overview of some definitional issues for sports injury surveillance. Sports Med. 24(3):157-163.

Finch, C.F., Finch, Valuri, G., and Ozanne-Smith, J. 1999. Injury surveillance during medical coverage of sporting events - development and testing of a standardized data collection form. J Sci Med Sport. 2:42-56.

Fuller C, Drawer S. 2004. The application of risk management in sport. Sports Med. 34:34956.

Fuller, C.W., Ekstrand, J., Junge, A., Andersen, T.E., Bahr, R., Dvorak, J., Hägglund, M., McCrory, P., and Meeuwisse, W.H. 2006. Consensus statement on injury definitions and data collection procedures in studies of football (soccer) injuries. Scand. J. Med. Sci. Sport. 16(2): 83-92.

Fuller CW, Ekstrand J, Junge A, et al. 2006. Consensus statement on injury definitions and data collection procedures in studies of football (soccer) injuries. Br J Sports Med. 40:193-201. 
Fuller, C.W., Molloy, M.G., Bagate, C., Bahr, R., Brooks, J.H., Donson, H., Kemp, S.P., McCrory, P., McIntosh, A.S., Meeuwisse, W.H., Quarrie, K.L., Raftery, M., and Wiley, P. 2007. Consensus Statement on Injury Definitions and Data Collection Procedures for Studies of Injuries in Rugby Union. Clin. J. Sport Med. 17(3): 177-181.

Fuller CW, Taylor A, Raftery M. 2015. Epidemiology of concussion in men's elite Rugby-7s (Sevens World Series) and Rugby-15s (Rugby World Cup, Junior World Championship and Rugby Trophy, Pacific Nations Cup and English Premiership). Br J Sports Med. 49(7):478-83.

van Gent, R.N., Siem, D., Van Middeloop, M., Van Os, A.G., Bierma-Zeinstra, S.M.A., and Koes, B.W. 2007. Incidence and determinants of lower extremity running injuries in long distance runners: A systematic review. Sport en Geneeskd. 40(4): 16-29.

Gessel, L.M., Fields, S.K., Collins, C.L., Dick, R.W., and Comstock, R.D. 2007. Concussions among United States high school and collegiate athletes. J Athl Train 42(4): 495-503.

Gosheger, G., Liem, D., Ludwig, K., Greshake, O., and Winkelmann, W. 2003. Injuries and overuse syndromes in golf. Am J Sport. Med 31(3): 438-443.

Hamilton, G.M., Meeuwisse, W.H., Emery, C. a., and Shrier, I. 2011. Subsequent Injury Definition, Classification, and Consequence. Clin. J. Sport Med. 21(6): 508-514.

Hinton, R.Y., Lincoln, A.E., and Almquist, J.L. 2005. Epidemiology of Lacrosse Injuries in High School - Aged Girls and Boys A 3-Year Prospective Study. Sport. Med. 33(9): 1305-1314.

Inklaar, H. 1994. Soccer injuries. I: Incidence and severity. Sports Med. 18:55-73.

Jackson, T.J., Starkey, C., McElhiney, D., and Domb, B.G. 2013. Epidemiology of hip injuries in the National Basketball Association: A 24-year overview. Orthop. J. Sport. Med. 1:1-7.

Junge, A. 2006. Injuries in Team Sport Tournaments During the 2004 Olympic Games. Am. J. Sports Med. 34(4):565-576.

Junge, A., Engebretsen, L., Alonso, J.M., Renström, P., Mountjoy, M., Aubry, M., and Dvorak, J. 2008a. Injury surveillance in multi-sport events: the International Olympic Committee approach. Br. J. Sports Med. 42:413-421.

Junge, A., Engebretsen, L., Alonso, J.M., Renström, P., Mountjoy, M., and Dvorak, J. 2008b. Injury surveillance in multi-sport events - the IOC approach. Br. J. Sports Med. 42(6): 413-421.

Junge, A., Engebretsen, L., Mountjoy, M.L., Alonso, J.M., Renström, P. A., Aubry, M.J., and Dvorak, J. 2009. Sports injuries during the Summer Olympic Games 2008. Am. J. Sports Med. 37(11):2165-72. 
Junge, A, and Dvorak, J. 2000. Influence of definition and data collection on the incidence of injuries in football. Am. J. Sports Med. 28(5 Suppl):S40-S46.

Junge, A., \& Dvorak, J. (2013). Injury surveillance in the world football tournaments 19982012. Br J Sports Med. 47:782-788.

Junge A, Dvorak J. 2015. Football injuries during the 2014 FIFA World Cup. Br J Sports Med. 49(9):599-602.

Kerr, H.A., Curtis, C., Micheli, L.J., Kocher, M.S., Zurakowski, D., Kemp, S.P.T., and Brooks, J.H.M. 2008. Collegiate rugby union injury patterns in New England: a prospective cohort study. Br. J. Sports Med. 42(7):595-603.

Kerr, Z.Y., Dompier, T.P., Snook, E.M., Marshall, S.W., Klossner, D., Hainline, B., and Corlette, J. 2014. National collegiate athletic association injury surveillance system: Review of methods for 2004-2005 through 2013-2014 data collection. J. Athl. Train. 49(4): 552560 .

Kerr, Z.Y., Kroshus, E., Grant, J., Parsons, J.T., Folger, D., Hayden, R., and Dompier, T.P. 2016. Epidemiology of national collegiate athletic association men's and women's crosscountry injuries, 2009-2010 through 2013-2014. J. Athl. Train. 51(1):57-64.

King, D.A., Gabbett, T.J., Gissane, C., and Hodgson, L. 2009. Epidemiological studies of injuries in rugby league: Suggestions for definitions, data collection and reporting methods. J. Sci. Med. Sport 12(1):12-19.

Ljungqvist, A., Jenoure, P., Engebretsen, L., Ljungqvist, A., Jenoure, P., Engebretsen, L., Alonso, J.M., Bahr, R., Clough, A., Bondt, G. De, Dvorak, J., Maloley, R., Matheson, G., Meeuwisse, W., Meijboom, E., Mountjoy, M., Pelliccia, A., Schwellnus, M., Sprumont, D., and Schamasch, P. 2009. The International Olympic Committee ( IOC ) Consensus Statement on periodic health evaluation of elite athletes March 2009. Br. J. Sports Med. 43(9):631-643.

Lynch, T.S., Saltzman, M.D., Ghodasra, J.H., Bilimoria, K.Y., Bowen, M.K., and Nuber, G.W. 2013. Acromioclavicular joint injuries in the national football league: epidemiology and management. Am. J. Sports Med. 41:2904-2908.

McKay, C., Tufts, R., Shaffer, B., and Meeuwisse, W. 2014. The epidemiology of professional ice hockey injuries:A prospective report of six National Hockey League seasons. Br. J. Sports Med. 48(1):57-62.

van Mechelen, W., Hlobil, H., and Kemper, H.C.G. 1992. Incidence, Severity, Aetiology and Prevention of Sports Injuries: A Review of Concepts. Sport. Med. An Int. J. Appl. Med. Sci. Sport Exerc. 14(2):82-99. 
Meeuwisse, W., H MD, P., Tyreman, H., Hagel, B., Emery, C., and BScPT, P. 2007. A Dynamic Model of Etiology in Sport Injury: The Recursive Nature of Risk and Causation. Clin J Sport Med. 17(3):215-219.

Meeuwisse, W.H. 1994. Assessing causation in sport injury: a multifactoral model. Clin J Sport Med. 4(3):166-170

Meeuwisse, W.H., and Love, E.J. 1997. Athletic injury reporting. Development of universal systems. Sports Med. 24:184 -204.

Meeuwisse, W.H., and Love, E.J. 1998. Development, implementation, and validation of the Canadian Intercollegiate Sport Injury Registry. Clin J Sport Med. 8:164 -177.

Meeuwisse, W.H., Hagel, B.E., Mohtadi, N.G., Butterwick, D.J., and Fick, G.H. 2000. The distribution of injuries in men's Canada West university football. A 5-year analysis. Am J Sport. Med. 28(4):516-523.

Meeuwisse, W.H., Sellmer, R., and Hagel, B.E. 2003. Rates and risks of injury during intercollegiate basketball. Am. J. Sports Med. 31(3): 379-385.

Moore, I.S., Ranson, C., and Mathema, P. 2015. Injury Risk in International Rugby Union: Three-Year Injury Surveillance of the Welsh National Team. Orthop. J. Sport. Med. 3(7):1-9

Morgan, R.J., Bankston, L.S., Hoenig, M.P., and Connor, P.M. 2010. Evolving management of middle-third clavicle fractures in the National Football League. Am. J. Sports Med. 38(10):2092-6.

Mountjoy, M., Junge, A., Alonso, J.M., Clarsen, B., Pluim, B.M., Shrier, I., van den Hoogenband, C., Marks, S., Gerrard, D., Heyns, P., Kaneoka, K., Dijkstra, H.P., and Khan, K.M. 2015a. Consensus statement on the methodology of injury and illness surveillance in FINA (aquatic sports). Br. J. Sports Med. 1:1-7.

Mountjoy, M., Junge, A., Alonso, J.M., Engebretsen, L., Dragan, I., Gerrard, D., Kouidri, M., Luebs, E., Shahpar, F.M., and Dvorak, J. 2010. Sports injuries and illnesses in the 2009 FINA World Championships (Aquatics). Br. J. Sports Med. 44:522-527.

Mountjoy M, Junge A, Benjamen S. et al. 2015. Competing with injuries: injuries prior to and during the 15th FINA World Championships 2013 (aquatics) Br J Sports Med. $49(1): 37-43$.

Olympic Charter. International Olympic Committee. Lausanne/Switzerland. September 2015. https://stillmed.olympic.org/Documents/olympic_charter_en.pdf (accessed 6 Mar 2017). 
Olympic Movement Medical Code. In force as from March 2016. https://stillmed.olympic.org/media/Document\%20Library/OlympicOrg/IOC/WhoWe-Are/Commissions/Medical-and-Scientific-Commission/Olympic-MovementMedical-Code-31-03-2016.pdf (accessed 6 Mar 2017).

OUA 2014-2020 Strategic Plan (2014). Retrieved from http://oua.prestosports.com/ima ges/2014-2020_Ontario_University_Athletics_Strategic_Plan.pdf (accessed March 30, 2017).

Pickett, W., Brison, R.J., Mackenzie, S.G., Garner, M., King, M. a, Greenberg, T.L., and Boyce, W.F. 2000. Youth injury data in the Canadian Hospitals Injury Reporting and Prevention Program: do they represent the Canadian experience? Inj. Prev. 6(1):9-15.

Podlog, L., Buhler, C.F., Pollack, H., Hopkins, P.N., and Burgess, P.R. 2015. Time trends for injuries and illness, and their relation to performance in the National Basketball Association. J. Sci. Med. Sport. 18(3):278-282.

Pollack, K.M., D’Angelo, J., Green, G., Conte, S., Fealy, S., Marinak, C., McFarland, E., and Curriero, F.C. 2016. Developing and implementing major league baseball's health and injury tracking system. Am. J. Epidemiol. 183(5):490-496.

Posner, M., Cameron, K.L., Wolf, J.M., Belmont, P.J., and Owens, B.D. 2011. Epidemiology of Major League Baseball injuries. Am. J. Sports Med. 39(8):1676-80.

Prien, A., Mountjoy, M., Miller, J., Boyd, K., van den Hoogenband, C., Gerrard, D., Cherif, M.Y., Lu, Y., Nanousis, K., Ortiz Liscano, E.I., Shahpar, F.M., and Junge, A. 2016. Injury and illness in aquatic sport: how high is the risk? A comparison of results from three FINA World Championships. Br. J. Sports Med. (August): bjsports-2016-096075.

Rechel, J.A., Yard, E.E., and Comstock, D. 2008a. An epidemiologic comparison of high school sports injuries sustained in practice and competition. J. Athl. Train. 43(2):197-204.

Rechel, J.A., Yard, E.E., and Comstock, R.D. 2008b. An epidemiologic comparison of high school sports injuries sustained in practice and competition. J. Athl. Train. 43(2):197204.

Ruedl, G., Schobersberger, W., Pocecco, E., Blank, C., Engebretsen, L., Soligard, T., Steffen, K., Kopp, M., and Burtscher, M. 2012. Sport injuries and illnesses during the first Winter Youth Olympic Games 2012 in Innsbruck, Austria. Br. J. Sports Med. 46:1030-1037.

Schick, D.M., and Meeuwisse, W.H. 2003. Injury rates and profiles in female ice hockey players. / Caracteristiques et taux des blessures chez des joueuses de hockey sur glace. Am. J. Sports Med. 31(1): 47-52.

Schwellnus M, Derman W, Jordaan E, et al. 2013. Factors associated with illness in 46 athletes participating in the London 2012 Paralympic Games: a prospective cohort study involving 49,910 athlete-days. Br J Sports Med. 47:433-40. 
Shankar, P.R., Fields, S.K., Collins, C.L., Dick, R.W., and Comstock, R.D. 2007. Epidemiology of High School and Collegiate Football Injuries in the United States, 2005 2006. Am. J. Sports Med. 35(8):1295-1303.

Smoljanovic, T., Bojanic, I., Hannafin, J.A., Hren, D., Delimar, D., and Pecina, M. 2011. Traumatic and overuse injuries among international elite junior rowers. Am. J. Sports Med.37(6):1193-9.

Soligard, T., Steffen, K., Palmer-Green, D., Aubry, M., Grant, M.E., Meeuwisse, W., Mountjoy, M., Budgett, R., and Engebretsen, L. 2015. Sports injuries and illnesses in the Sochi 2014 Olympic Winter Games. Br J Sport. Med. 49(7):441-447.

Starkey, C. 2000. Injuries and Illnesses in the National Basketball Association: A 10-Year Perspective. J. Athl. Train. 35(2):161-167.

Thompson, S.H., Smith, P. and DiGioacchino, R., 2004. Performance-related injuries and exercise orientation of National Collegiate Athletic Association Division I, II, and III female collegiate cross country runners. WSPAJ. 13(2):17-26.

Timpka, T., Alonso, J.-M., Jacobsson, J., Junge, A., Branco, P., Clarsen, B., Kowalski, J., Mountjoy, M., Nilsson, S., Pluim, B., Renström, P., Rønsen, O., Steffen, K., and Edouard, P. 2014a. Injury and illness definitions and data collection procedures for use in epidemiological studies in Athletics (track and field): consensus statement. Br. J. Sports Med. 48(7):483-90.

Timpka, T., Jacobsson, J., Bickenbach, J., Finch, C.F., Ekberg, J., and Nordenfelt, L. 2014b. What is a sports injury? Sports Med. 44(4):423-428.

Tuominen, M., Stuart, M.J., Aubry, M., Kannus, P., and Parkkari, J. 2015. Injuries in men's international ice hockey: a 7-year study of the International Ice Hockey Federation Adult World Championship Tournaments and Olympic Winter Games. Br. J. Sports Med. 49(1):30-36.

What is the NCAA? (2015). Retrieved from http://www.ncaa.org/about/resources/mediacenter/ncaa-101/what-ncaa (accessed March 6, 2017).

Willick SE, Webborn N, Emery C, et al. 2013. The epidemiology of injuries at the London 66 2012 Paralympic Games. Br J Sports Med. 47:426-32.

Yang, J., Tibbetts, A.S., Covassin, T., Cheng, G., Nayar, S., and Heiden, E. 2012. Epidemiology of overuse and acute injuries among competitive collegiate athletes. J. Athl. Train. 47(2):198-204. 


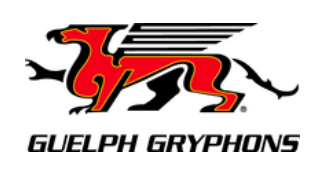

Sport:

\section{Athletic Therapist / Trainers' Weekly Report on Injuries and IIInesses}

Week of Report:
UNIVERSITY GUELPH

HPC 度

Contact Details (email/tel.):

Please report: (1) All injuries (traumatic and overuse) and (2) all illnesses newly incurred in competition or training during the athletic season regardless of the consequences with respect to absence from competition or training. The information provided is for medical and research purposes and will be treated confidentially.

1. Injury - Example

\begin{tabular}{|c|c|c|c|c|c|c|c|}
\hline \multirow[t]{2}{*}{ Identifier } & \multirow{2}{*}{$\begin{array}{l}\text { age } \\
22 \\
\text { injury body part, side } \\
\text { thumb, left }\end{array}$} & \multirow{2}{*}{$\begin{array}{l}\text { gender } \\
F \\
\text { code } \\
17\end{array}$} & \multicolumn{2}{|c|}{$\begin{array}{l}\text { sport and event } \\
\text { women's hockey }\end{array}$} & \multicolumn{2}{|c|}{$\begin{array}{l}\text { in competition or training } \\
\text { playoffs - round } 1 \text {, game } 1\end{array}$} & \multirow{2}{*}{$\begin{array}{l}\text { date \& time } \\
24 / \mathrm{Feb} / 16-20: 00 \\
\text { absence in days } \\
14\end{array}$} \\
\hline & & & $\begin{array}{l}\text { type of injury } \\
\text { sprain }\end{array}$ & $\begin{array}{l}\text { code } \\
8\end{array}$ & $\begin{array}{l}\text { cause of injury } \\
\text { on-ice collision }\end{array}$ & $\begin{array}{l}\text { code } \\
11\end{array}$ & \\
\hline
\end{tabular}

\begin{tabular}{|l|l|l|l|l|l|l|l|}
\hline Identifier & age & gender & \multicolumn{2}{|l|}{ sport and event } & \multicolumn{2}{|l|}{ round / heat or training } & \multicolumn{2}{l|}{ date \& time } \\
\cline { 2 - 7 } & injury body part, side & code & type of injury & code & cause of injury & code & absence in days \\
\hline
\end{tabular}

\begin{tabular}{|l|l|l|l|l|l|l|l|}
\hline Identifier & age & gender & \multicolumn{2}{l|}{ sport and event } & \multicolumn{2}{l|}{ round / heat or training } & \multicolumn{2}{|l|}{ date \& time } \\
\cline { 2 - 7 } & injury body part, side & code & type of injury & code & cause of injury & code & absence in days \\
\hline
\end{tabular}

\begin{tabular}{|l|l|l|l|l|l|l|l|}
\hline Identifier & age & gender & \multicolumn{2}{|l|}{ sport and event } & \multicolumn{2}{l|}{ round / heat or training } & \multicolumn{2}{l|}{ date \& time } \\
\cline { 2 - 7 } & injury body part, side & code & type of injury & code & cause of injury & code & absence in days \\
\hline
\end{tabular}

\begin{tabular}{|l|l|l|l|l|l|l|l|}
\hline Identifier & age & gender & \multicolumn{2}{|l|}{ sport and event } & \multicolumn{2}{|l|}{ round / heat or training } & \multicolumn{2}{l|}{ date \& time } \\
\cline { 2 - 7 } & injury body part, side & code & type of injury & code & cause of injury & code & absence in days \\
\hline
\end{tabular}

2. Illness - Example

\begin{tabular}{|c|c|c|c|c|c|c|c|}
\hline \multirow[t]{2}{*}{ Identifier } & \multirow{2}{*}{$\begin{array}{l}\text { age } \\
23 \\
\text { affected system } \\
\text { upper respiratory tract }\end{array}$} & \multirow{2}{*}{$\begin{array}{l}\text { gender } \\
M \\
\text { code } \\
1 \\
\end{array}$} & \multicolumn{2}{|l|}{$\begin{array}{l}\text { sport and event } \\
\text { basketball }\end{array}$} & \multicolumn{2}{|l|}{$\begin{array}{l}\text { diagnosis } \\
\text { tonsillitis, cold }\end{array}$} & \multirow{2}{*}{$\begin{array}{l}\text { occurred on (date) } \\
22 / \text { Feb/16 } \\
\text { absence in days } \\
3\end{array}$} \\
\hline & & & $\begin{array}{l}\text { main symptom(s) } \\
\text { pain, fever }\end{array}$ & $\begin{array}{l}\text { code } \\
1,2\end{array}$ & $\begin{array}{l}\text { cause of illness } \\
\text { infection }\end{array}$ & $\begin{array}{l}\text { code } \\
2\end{array}$ & \\
\hline \multirow[t]{2}{*}{ Identifier } & age & gender & \multicolumn{2}{|l|}{ sport and event } & \multicolumn{2}{|l|}{ diagnosis } & occurred on (date) \\
\hline & affected system & code & main symptom(s) & code & cause of illness & code & absence in days \\
\hline \multirow[t]{2}{*}{ Identifier } & age & gender & \multicolumn{2}{|l|}{ sport and event } & \multicolumn{2}{|l|}{ diagnosis } & occurred on (date) \\
\hline & affected system & code & main symptom(s) & code & cause of illness & code & absence in days \\
\hline \multirow[t]{2}{*}{ Identifier } & age & gender & \multicolumn{2}{|l|}{ sport and event } & \multicolumn{2}{|l|}{ diagnosis } & occurred on (date) \\
\hline & affected system & code & main symptom(s) & code & cause of illness & code & absence in days \\
\hline \multirow[t]{2}{*}{ Identifier } & age & gender & \multicolumn{2}{|l|}{ sport and event } & \multicolumn{2}{|l|}{ diagnosis } & occurred on (date) \\
\hline & affected system & code & main symptom(s) & code & cause of illness & code & absence in days \\
\hline
\end{tabular}




\section{Codes and Classifications}

For Injuries

\begin{tabular}{|c|c|c|}
\hline \multicolumn{3}{|c|}{ Injured body part (location of injury) } \\
\hline Head and Trunk & Upper extremity & Lower extremity \\
\hline 1 - head (specify: eye, ear, nose, face, dental) & 11 - shoulder (incl. clavicle) & $21-$ hip/groin \\
\hline $\mathbf{2}$ - neck / cervical spine & 12 - upper arm & 22 - thigh \\
\hline $\mathbf{3}$ - chest / sternum / ribs & 13 - elbow & 23 - knee \\
\hline 4-thoracic spine / upper back & 14 - forearm & $\mathbf{2 4 - l o w e r ~ l e g ~ / ~ A c h i l l e s ~ t e n d o n ~}$ \\
\hline 5 - lumbar spine / lower back & $15-$ wrist & $25-$ ankle \\
\hline 6 - abdomen & 16 - hand & $26-$ foot / toe \\
\hline 7 - pelvis / sacrum / buttock & 17 - finger / thumb & 27 - other \\
\hline
\end{tabular}

\begin{tabular}{|c|c|c|}
\hline \multicolumn{3}{|c|}{ Type of injury (diagnosis) } \\
\hline 1 - concussion (regardless of loss of consciousness) & $\mathbf{8}$ - sprain (injury of joint and/or ligaments) & 15 - tendinosis / tendinopathy \\
\hline 2 - fracture (traumatic) & $\mathbf{9}$ - lesion of meniscus or cartilage & 16 - arthritis / synovitis / bursitis \\
\hline 3 - stress fracture (overuse) & 10 - strain / muscle rupture / tear & 17 - fasciitis / aponeurosis injury \\
\hline 4 - other bone injuries & 11 - contusion / haematoma / bruise & 18 - impingement \\
\hline 5 - dislocation, subluxation & 12 - laceration / abrasion / skin lesion & 19 - growth plate disturbance / avulsion \\
\hline 6 - tendon rupture & 13 - nerve injury / spinal cord injury & 20 - muscle cramps or spasm \\
\hline 7 - ligamentous & 14 - dental injury / broken tooth & 21 - other \\
\hline
\end{tabular}

\begin{tabular}{|c|c|c|}
\hline \multicolumn{3}{|c|}{ Cause of injury } \\
\hline 1 - overuse (gradual onset) & 11 - contact with another athlete & 21 - field of play conditions \\
\hline 2 - overuse (sudden onset) & 12 - contact: moving object (e.g. ball) & 22 - weather condition \\
\hline 3 - non-contact trauma & 13 - contact: stagnant object (e.g. wall) & 23 - equipment failure \\
\hline 4- recurrence of previous injury & 14 - violation of rules (foul play) & $\mathbf{2 4}$ - other \\
\hline
\end{tabular}

Estimated duration of absence from training and competition (in days)

Please provide an estimate of the number of days that the athlete will not be able to undertake his/her normal training program or will not be able to compete.
$\mathbf{0}=0$ days
7 = 1 week
$1=1$ day
$14=2$ weeks
$\mathbf{2}=2$ days
$\mathbf{2 1}=3$ weeks
$28=4$ weeks
$>\mathbf{3 0}=$ more than 4 weeks
$>\mathbf{1 8 0}=6$ months or more

For Illnesses

\begin{tabular}{|c|c|c|c|}
\hline \multicolumn{4}{|c|}{ Affected system } \\
\hline \multirow{2}{*}{\multicolumn{2}{|c|}{$\begin{array}{l}\mathbf{1} \text { - upper respiratory tract (nose, sinuses, pharynx, larynx) } \\
\mathbf{2} \text { - lower respiratory tract (trachea, bronchi, lungs) }\end{array}$}} & 6-metabolic / endocrinological & 11 - ophthalmological / ontological \\
\hline & & 7 - allergic / immunological & 12 - dental \\
\hline \multirow{2}{*}{\multicolumn{2}{|c|}{$\begin{array}{l}3 \text { - gastro-intestinal } \\
\mathbf{4} \text { - cardio-vascular }\end{array}$}} & 8 - haematological & 13 - neurological / CNS \\
\hline & & 9 -dermatologic & 14 - psychiatric / psychological \\
\hline \multicolumn{2}{|c|}{ 5-uro-genital/gynaecological } & 10 - musculo-skeletal & 15 - other \\
\hline \multicolumn{4}{|c|}{ Main symptom(s) } \\
\hline 1-pain / ache / soreness & 6-irregul & eartbeat / palpitation & 11 - fainting / syncope \\
\hline 2 - fever / chills & 7 - dehydr & n / excess sweating & 12 - numbness / weakness / tingling \\
\hline 3 - nausea, vomiting, diarrhoea & $8-\operatorname{rash} / i$ & / eczema & 13 - fatigue / lack of energy / lethargy \\
\hline 4 - cough, wheezing, dyspnoea & 9- nasal c & estion / rhinorrhoea & 14 - sleep disturbance \\
\hline 5 - shortness of breath & $10-$ dizzin & / vertigo & $\begin{array}{l}\mathbf{1 5} \text { - psychol. Problems / anxiety / depression } \\
\mathbf{1 6} \text { - other }\end{array}$ \\
\hline \multicolumn{4}{|c|}{ Cause of illness/symptom(s) } \\
\hline \multirow{2}{*}{$\begin{array}{l}\mathbf{1} \text { - pre-existing (e.g. asthma, allergy) } \\
\mathbf{2} \text { - infection }\end{array}$} & \multicolumn{2}{|c|}{ 3- exercise-induced } & $\mathbf{5}$ - reaction to medication \\
\hline & 4 - en & nmental & 6 - other \\
\hline
\end{tabular}




\section{APPENDIX 2: Athletic Therapists'/Student Trainers' Individual Injury and Illness Report}

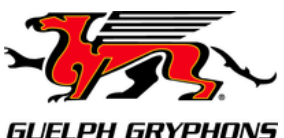

Identifier:

\section{Athletic Therapist / Student Trainer Report Form University varsity athlete health surveillance throughout the season}

Sport:
Reporting week:

UNIVERSITY

Exposure in training (in minutes per day)

Please indicate day of injury by circling one of the days below.

no training in past 7 days

\begin{tabular}{|l|l|l|l|l|l|l|l|}
\hline & Monday & Tuesday & Wednesday & Thursday & Friday & Saturday & Sunday \\
\hline Full & & & & & & & \\
\hline Limited & & & & & & & \\
\hline
\end{tabular}

Exposure in competition:

$\square$ no competition in past 7 days

Total days present at a competition:

Event competed in:

/ number of starts or matches:

Event competed in: / number of starts or matches:

1. Has the athlete had any difficulties in participating in normal training and competition due to injury,

illness or other health problems during the past 7

days?

a full participation without health problems

full participation but with injury / illness

a reduced participation due to injury / illness

$\square$ could not participate due to injury / illness

3. How much was the athlete's performance affected

by injury, illness or other health problems during the

past 7 days?

$\square$ not at all

$\square$ minor

$\square$ moderate

$\square$ major

$\square$ could not participate at all
2. How much did the athlete modify the volume, content or intensity of training due to injury, illness or other health problems during the past 7 days?

$\square$ no modification of training

$\square$ minor

$\square$ moderate

$\square$ major

$\square$ no training

4. How severe were the athlete's health problems /

complaints during the past 7 days?

$\square$ no symptoms / health problems

$\square$ minor

$\square$ moderate

口 severe

$\square$ no training an no competition

If the athlete had complaints or any injury, illness or other health problems, please answer the following questions:

5. On how many days did the athlete have complaints
during the past 7 days?
7. Are the complaints new, recurrent or have been
reported last week?
$\square$ new
$\square$ recurrent after full participation in sport
$\square$ reported last week

6 . How many training or competition days did the

athlete miss due to these complaints?

\begin{tabular}{|ll|}
\hline 8. How were the complaints treated in the last 7 days? (check all that apply) \\
\hline$\square$ no treatment & $\square$ pharmacological \\
$\square$ rest & $\square$ surgical \\
$\square$ massage & $\square$ physiotherapy \\
$\square$ athletic therapy & $\square$ others, specify \\
\hline
\end{tabular}




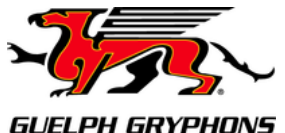

Identifier:
Athlete Report Form University varsity athlete health surveillance throughout the season

Sport:
Reporting week:
UNIVERSITY of GUELPH

HPC

Please answer the following questions to provide information on the severity of your injury/illness:

\begin{tabular}{|c|c|}
\hline $\begin{array}{l}\text { 1. Have you had any difficulties in participating in normal } \\
\text { training and competition due to injury, illness or other } \\
\text { health problems during the past } 7 \text { days? } \\
\text { full participation without health problems } \\
\text { full participation but with injury / illness } \\
\text { reduced participation due to injury / illness } \\
\square \text { could not participate due to injury / illness }\end{array}$ & $\begin{array}{l}\text { 2. To what extent have you modified the volume, content or } \\
\text { intensity of training due to injury, illness or other health } \\
\text { problems during the past } 7 \text { days? } \\
\text { no modification of training } \\
\text { to a minor extent } \\
\text { to a moderate extent } \\
\text { to a major extent } \\
\square \text { could not participate at all }\end{array}$ \\
\hline $\begin{array}{l}\text { 3. To what extent have injury, illness or other health } \\
\text { problems affected your performance during the past } 7 \text { days? } \\
\text { no effect } \\
\text { to a minor extent } \\
\text { to a moderate extent } \\
\text { to a major extent } \\
\text { could not participate at all }\end{array}$ & $\begin{array}{l}\text { 4. To what extent have you experienced symptoms / health } \\
\text { problems during the past } 7 \text { days? } \\
\text { no symptoms / health problems } \\
\text { to a minor extent } \\
\text { to a moderate extent } \\
\text { to a severe extent }\end{array}$ \\
\hline
\end{tabular}

If you had complaints or any injury, illness or other health problems, please also answer the following questions:

\begin{tabular}{|l|l|}
\hline $\begin{array}{l}5 . \text { On how many days have you had complaints during the } \\
\text { past } 7 \text { days? }\end{array}$ & $\begin{array}{l}\text { 6. How many training or competition days did you miss due } \\
\text { to these complaints? }\end{array}$ \\
\hline $\begin{array}{l}\text { 7. Are the complaints new, recurrent or have been reported } \\
\text { last week? }\end{array}$ & \\
$\begin{array}{l}\text { new } \\
\text { recurrent after full participation in sport }\end{array}$ & \\
& \\
& reported last week
\end{tabular}

\begin{tabular}{|llll|}
\hline 8. Please indicate the location of the complaints referred to above. & \\
\hline$\square$ head, specify: eye, ear, nose, face, dental & $\square$ shoulder / clavicle / scapula & $\square$ & hip/groin \\
$\square$ neck / cervical spine & $\square$ upper arm & $\square$ thigh \\
$\square$ chest / sternum / rib & $\square$ elbow & $\square$ knee \\
$\square$ thoracic spine / upper back & $\square$ forearm & $\square$ lower leg / Achilles tendon \\
$\square$ lumbar spine / lower back & $\square$ wrist & $\square$ ankle \\
$\square$ abdomen & $\square$ hand & $\square$ foot / toe \\
$\square$ pelvis / sacrum / buttock & $\square$ finger / thumb & $\square$ other, please specify \\
\hline
\end{tabular}

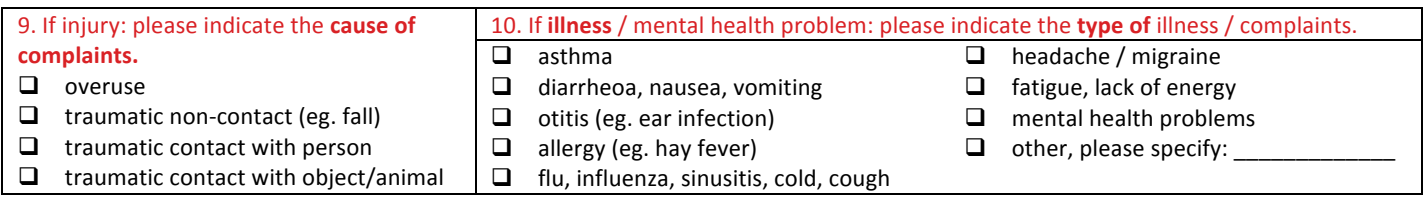

11. If a trainer / clinician / healthcare professional made a diagnosis, please report: 